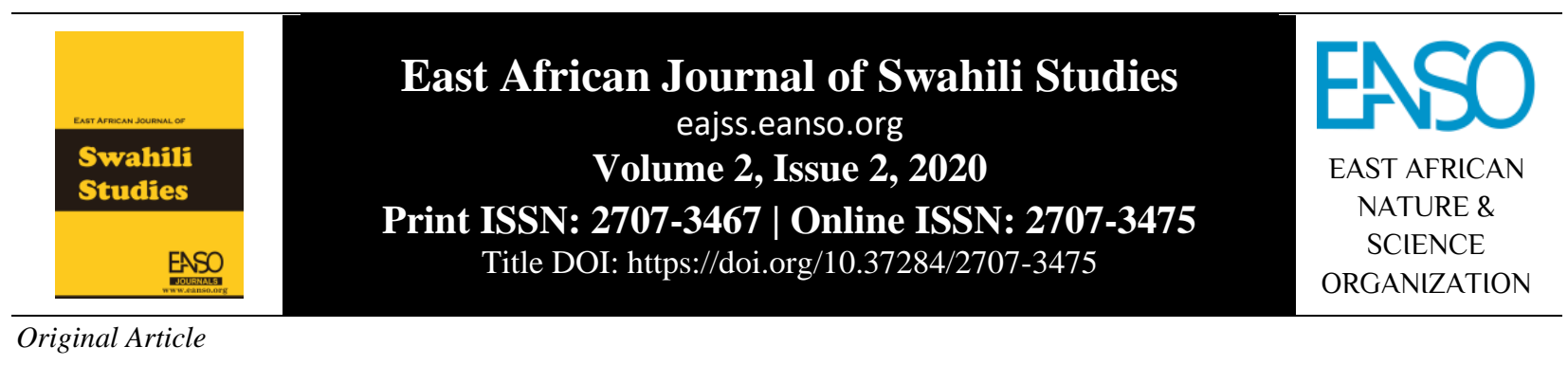

\title{
Ruwaza za Toni katika Vitenzi Visoukomo Changamani vya Kirombo
}

Peter T. Mramba ${ }^{1 *}$

${ }^{1}$ Chuo Kikuu cha Mt. Augustino, S. L. P. 307, Malimbe-Mwanza, Tanzania.

*Barua pepe ya mawasiliano: mrambadoctor@gmail.com.

DOI ya Nakala: https://doi.org/10.37284/eajss.2.2.242

\section{Tarehe ya Uchapishaji: IKISIRI}

30 Novemba 2020 Makala haya yanahusu uchanganuzi wa ruwaza za Toni katika Vitenzi visoukomo changamani vya Kirombo. Lengo kuu la makala haya ni

Istilahi Muhimu: kuchunguza ruwaza ya ujitokezaji wa toni katika vitenzi visoukomo

Sifa za Kimuundo, changamani vya Kirombo na kanuni zinazotawala utokeaji huo. Aidha,

Kimofolojia,

Kisintaksia,

Wizani,

Ushairi Huru.

malengo mahususi: kubainisha silabi inayohusishwa na Tonijuu Msingi (kuanzia sasa TJM) na mbili, kujadili ruwaza ya utokeaji wa toni katika vitenzi visoukomo changamani vya Kirombo na kanuni zinazotawala hutokeaji huo. Data ya Makala haya imetokana na utafiti mpana uliofanywa juu ya toni katika wilaya ya Rombo (2019) ambao uliongozwa na Nadharia ya Fonolojia Vipandesauti Huru kwa kutumia Mkabala wa Tonijuu Msingi kama ulivyoasisiwa na Goldsmith (1976) na kuboreshwa na wanazuoni mbalimbali katika taaluma ya fonolojia. Aidha, mbinu za ukusanyaji data zilizotumika ni pamoja na mahojiano na ushuhudiaji. Katika mahojiano vitenzi visoukomo changamani viliandaliwa kwa Kiswahili na watoataarifa walihitajika kuvitamka kwa Kirombo huku mtafiti akirekodi na kualamisha toni katika vitenzi hivyo. Matokeo ya utafiti yanaonesha kuwa kuna ruwaza mbalimbali za kitoni zinazoleteleza utokeaji wa kanuni za kitoni ambazo hutofautiana kutegemeana na idadi ya silabi na uwapo au kutowapo kwa yambwa. Aidha, vitenzi visoukomo changamani vya silabi moja hutawaliwa na kanuni ya uhusishaji wa toni chini na vitamkwa na sharti la ukubalifu, wakati vitenzi visoukomo changamani vya silabi mbili hadi ama tano au sita za shina hutawaliwa na kanuni ya udondoshaji wa TJM pamoja na kanuni nyingine kitoni kama vile: kanuni ya msambao wa tonijuu kuelekea kulia mwa shina, uhamaji wa TJM kutoka silabi ya kwanza kwenda katika silabi ya pili ya shina, unakiliji wa TJM katika silabi ya mwisho kasoro moja. Aidha, TJM hupachikwa katika mofimu ya yambwa kwa vitenzi visoukomo changamani vya silabi moja ya shina. Pia, matokeo yanaonesha kwamba kuna TJM mbili, 
yaani, ya kwanza ni ya yambwa na ya pili ikiwa ya shina, ambapo katika mchakato wa ukokotozi wa uibuzi wa toni, TJM ya shina hudondoshwa kwa kuwa yambwa ina nguvu zaidi. Makala haya yamesaidia kujua namna toni zinavyojitokeza katika vitenzi visoukomo changamani vya Kirombo kwa ujumla.

\section{APA CITATION}

Mramba, P. (2020). Ruwaza za Toni katika Vitenzi Visoukomo Changamani vya Kirombo. East African Journal of Swahili Studies, 2(2), 168-196. https://doi.org/10.37284/eajss.2.2.242

\section{CHICAGO CITATION}

Mramba, Peter. 2020. "Ruwaza za Toni katika Vitenzi Visoukomo Changamani vya Kirombo". East African Journal of Swahili Studies 2 (2), 168-196. https://doi.org/10.37284/eajss.2.2.242.

\section{HARVARD CITATION}

Mramba, P. (2020) "Ruwaza za Toni katika Vitenzi Visoukomo Changamani vya Kirombo", East African Journal of Swahili Studies, 2(2), pp. 168-196. doi: 10.37284/eajss.2.2.242.

\section{IEEE CITATION}

P. Mramba, "Ruwaza za Toni katika Vitenzi Visoukomo Changamani vya Kirombo", EAJSS, vol. 2, no. 2, pp. 168-196, Nov. 2020.

\section{MLA CITATION}

Mramba, Peter. 2020. "Ruwaza za Toni katika Vitenzi Visoukomo Changamani vya Kirombo". East African Journal of Swahili Studies, Vol. 2, no. 2, Nov. 2020, pp. 168-196, doi:10.37284/eajss.2.2.242.

\section{UTANGULIZI}

Lugha za binadamu ulimwenguni zina sifa ya kuwa na vipambasauti (Massamba, 2011). Hivi ni vipengele vya uchanganuzi arudhi viwezavyo kuandamishwa kwenye vitamkwa. Vipengele hivi ni pamoja na toni, mkazo, usilabi, kidatu, unazali, unguvunguvu, na nguvumsikiko. Makala haya yanahusika na kipengee cha toni ambacho ni kipambasauti chenye uamilifu wa kileksika na kisarufi kinachoweza kupambanua/kutofautisha maana za maneno na kuwakilisha njeo (Odden, 2005; Massamba, 2011: 171; Batibo, 2012). Kwa mujibu wa wataalamu Heine na Nurse (2000), Nurse na Philipson (2003) na Batibo (2012), lugha nyingi za Kiafrika zina toni, ukiondoa zile za kundi la lugha za Kihamitiki kama vile Kishemu/Kihamu. Toni ni miongoni mwa sifa za kiarudhi ${ }^{1}$ zilizowahamasisha wanaisimu kuchunguza vipengele mbalimbali vya lugha za Kibantu kwa kutumia Nadharia za taaluma ya fonolojia (Hyman, 2008). Suala la uwakilishi wa toni katika lugha lilishamiri zaidi hasa pale mwanaisimu Goldsmith (1976) alipoonesha mwanga wa uchanganuzi wa

\footnotetext{
${ }^{1}$ Arudhi ni sifa inayohusu mabadiliko ya vipambasauti kama vile kidatu, nguvumsikiko, tempo na lahani (Massamba, 2012).
}

toni katika lugha mahususi kwa kutumia Nadharia ya Fonolojia Vipandesauti Huru kama maboresho ya Nadharia ya Fonolojia Zalishi (Hayman, 2003: 4-6 na Massamba, 2011: 178).

Kiisimuhistoria, wakati wa Bantu-mame lugha za Kibantu zilikuwa na toni, na kwamba toni zake zilikuwa sahili zaidi. Kutokana na mwachano wa lugha hizi na mabadiliko mengi ya kiisimu, imeleteleza kuwa na makundi manne ya lugha za Kibantu, ambayo ni: lugha zenye toni asilia kama vile Kimeetto-Makuwa na Kikurya, lugha zenye mkazo kama vile Kiswahili, lugha zenye viinitoni vinavyochombeza toni kama vile Kimochi na Kikonongo, na lugha ambazo zipo kati ya kuwa na kiinitoni na mkazo kama vile Kinyakyusa. Hata hivyo, lugha zenye toni zimewekwa katika makundi makuu mawili, ambayo ni lugha zenye toni asilia na lugha zenye viinitoni (Massamba, 2011: 176).

Aidha, lugha zenye toni asilia zina toni katika maumbo yake ya ndani na toni zake hazitabiriki. Lugha zenye toni zinazochombezwa na kiinitoni hazina toni katika maumbo yake ya ndani bali 
viinitoni na toni zake hutabirika na huwa na kiimbotoni msingi ambacho huhusishwa na kiinitoni kwenye silabi inayobeba kiinitoni hicho (Massamba, 2011: 177). Lugha zisizo na toni ni zile ambazo hazitumii kidatu kubainisha maana kileksika au kisarufi na aghalabu huwa na mkazo.

\section{LUGHA YA KIROMBO}

Kirombo ni lugha ya Kibantu iliyopo miongoni mwa jamiilugha za Kichaga. Kichaga hakina lugha moja inayoweza kuunganisha jamii ya Wachaga wote. Kila eneo (tarafa) lina lugha yake na kila kata ndani ya tarafa ina matamshi yake (Mramba, 2015). Kwa mujibu wa MLUTA (2009), jamii ya Wachaga ina jumla ya lugha asilia sita zinazotumika kwa mawasiliano ndani na nje ya eneo kitovu (Kilimanjaro). Lugha hizo ni Kirombo, Kimachame, Kikahe, Kihusa, Kimochi na Kisia. Hata hivyo, kuna lahaja zinazozungumzwa ndani ya jamii ya Wachaga kama vile Kiuru, Kiwoso, Kivunjo, Kichasu, Kimarangu na Kihanjo.

Kwa mujibu wa MLUTA (2009: xi), lugha za Kichaga zinapatikana mkoani Kilimanjaro na zimegawanywa katika makundi makuu manne ambayo ni: Kilimanjaro Kaskazini, Kusini, Mashariki na Magharibi, ambapo Kirombo kipo katika kundi la tatu ambalo ni Kilimanjaro Mashariki, lenye kujumuisha lugha za Kirombo na Kivunjo. Pia, lugha ya Kirombo imepewa utambulisho wa kanda E.623 kwa kuzingatia kigezo cha msingi wa kijiografia.

Kwa mujibu wa Sensa ya Watu na Makazi (2012), Rombo ina jumla ya Warombo 400,786 ambapo wanaume ni 197, 279 na wanawake 203,507 (Kitengo cha Takwimu Wilaya ya Rombo (KTWIRO)), ikiwa na maana kwamba, hiyo ni jumla ya idadi ya wakazi wa Wilaya ya Rombo kwa kipindi hicho ingawa hadi wakati utafiti huu unafanyika idadi hiyo bila shaka itakuwa imeongezeka kwa kiasi fulani ambacho hakiwezi kubainishwa wazi hadi hapo sensa nyingine itakapofanyika tena. Hata hivyo, kwa mujibu wa MLUTA (2009), kuna jumla ya Warombo 363, 576, wanaoishi kwenye miteremko ya Mlima Kilimanjaro katika Wilaya ya Rombo iliyopo mpakani mwa Kenya upande wa Kaskazini Mashariki na Wilaya ya Moshi Vijijini kwa upande wa Kusini. Katika Wilaya ya Rombo, eneo kitovu cha Warombo ni Kata ya Mengwe. Eneo hili limepakana na Kata ya Ngoyoni upande wa Mashariki, Magharibi limepakana na Msitu wa Hifadhi ya Mlima Kilimanjaro, Kaskazini limepakana na Kata ya Manda Juu na Manda Chini na Kusini kuna Kata za Mamsera Juu, Kati, na Chini (Mramba, 2015).

Kutokana na tafiti zilizofanywa na wataalamu mbalimbali kama Raum (1964), Polomé (1971), Van Spaandock (1971), Nurse na Philipson (1977), Heine na Nurse (2003), Martinet (2005), McHugh (2006), na Nelson (2013) katika lugha za Kichaga kama vile Kiold Mochi, Kimachame, Kivunjo, Kikahe na Kisiha, wamebaini kuwa lugha hizo zina toni. Aidha, wamebaini mabadiliko ya utokeaji wa ruwaza za toni katika lugha hizo hayapo sawa jambo linalofanya baadhi ya lugha kuwa na zaidi ya $66 \%$ ya tonichini na chini ya $34 \%$ ya tonijuu katika maneno yake. Kulingana na matokeo ya tafiti hizo na ikizingatiwa kwamba Kirombo ni miongoni mwa jamii lugha za Kichaga, Kirombo pia kina toni ingawa haikuelezwa ruwaza yake ya utokeaji ikoje hususani kwa vitenzi visoukomo changamani.

Aidha, Kirombo kinadhihirisha wazi kina toni kulingana na ruwaza za maneno yake ambayo kiothografia huonekana sawa ilhali wazawa wa lugha hiyo wanapoyatumia katika mazungumzo yao huchomoza maana tofauti. Tutazame mfano (1) hapa chini:

$\begin{aligned} \text { 1. a) ílya } & \text { 'kula' } \\ \text { Ilyá } & \text { 'kukataa' } \\ \text { b) íla } & \text { 'kulala' } \\ \text { ilá } & \text { 'kuita' }\end{aligned}$

Hivyo, katika 1(a) tunaona mambo yafuatayo: tonijuu ikijitokeza katika silabi ya kwanza ya neno na maana yake huwa ' $k u l a$ ' na maana hubadilika na kuwa 'kukataa' toni inapojitokeza katika silabi ya pili ya neno. Hali hiyo inajitokeza katika 1(ii) kama inavyoonekana hapo juu. Pia, toni katika Kirombo hubadili kategoria ya maneno kama inavyoonekana katika mfano wa 2:
2. a) i-kerésa
gereza $(\mathrm{N})$ i-kéresa chonga kitu(T)
b) sháa nje $(\mathrm{N})$ shaá
c) i-sháa beza /kataa (T) i-shaá kuja (T) ukucha mkubwa $(\mathrm{N})$ 
d) i- káa kaa la moto i-kàa kukaa/kuishi mahala fulani (T)

Katika 2 (a) tunaona mambo yafuatayo: tonijuu ikijitokeza katika silabi ya pili ya shina la neno huwa na maana ya gereza $(\mathrm{N})$, wakati ikihamia silabi ya kwanza ya shina huwa na maana ya chonga kitu (T). Halikadhalika, katika (b) tonijuu ikiwa katika irabu ya kwanza ya shina huwa na maana ya nje (N) na ikihamia kwenye silabi ya pili huwa na maana ya beza/ kataa (T). Hali hiyo inajitokeza pia katika mfano wa (c), ila katika mfano wa (d) tonichini ikitokea mahala pa tonijuu pia maana na kategoria hubadilika kama inavyoonekana.

Hii inatokana na ukweli kwamba lugha nyingi za Kibantu zina toni hususani zile za kundi la NaijaKongo ambapo pia Kirombo hupatikana humo (taz. Nurse na Philipson, 2003). Batibo (2012) akimnukuu Van Spaandock (1971), anasema, kiasili jamiilugha za Kichaga zina toni, ambapo katika kila silabi na kila neno huwa na toni maalum. Kutokana na maelezo hayo tunajiuliza: je, TJM katika vitenzi visoukomo changamani huhusishwa na silabi ipi? Je, ruwaza ya toni katika vitenzi visoukomo changamani katika Kirombo ikoje na hutawaliwa na kanuni zipi? Maswali haya na mengine mengi tuliyokuwa tukijiuliza, yamejibiwa vyema katika sehemu ya 3.0 ambapo ruwaza za ujitokezaji wa toni kuanzia silabi chache hadi nyingi kiidadi za mashina ya vitenzi visoukomo changamani zimechanganuliwa.

\section{RUWAZA YA UJITOKEZAJI WA TONI KATIKA VITENZI VISOUKOMO CHANGAMANI VYA KIROMBO}

Baada ya usuli huo, sasa tunaweza kuchunganua ruwaza ya ujitokezaji wa toni katika vitenzi visoukomo changamani kwa kuanza na vile vyenye idadi ndogo ya silabi hadi vyenye silabi nyingi za shina. Ingekuwa jambo la kheri kama tungefafanua lau kwa ufupi yambwa ni nini, na katika vitenzi visoukomo vya Kirombo hujitokeza sehemu gani, kabla ya kuangazia uchanganuzi wa uibuzi wa toni katika vitenzi visoukomo changamani.

Kwa mujibu wa Massamba, (2016:168) anafasili yambwa kuwa ni kipashio cha sentensi ambacho huelekezwa tendo au lengo. Kutokana na fasili hiyo, yambwa hujidhihirisha kupitia maumbo ya nafsi yanayojitokeza katika sentensi husika. Katika Kirombo nafsi imegawanywa katika makundi matatu ambayo ni: nafsi ya kwanza, nafsi ya pili na nafsi ya tatu. Nafsi hizi huwakilishwa kwa maumbo mbalimbali yanayojitokeza katika hali ya umoja na wingi kama inavyoonekana katika jedwali la 1.

\section{Viambishi vya Nafsi na Yambwa Kiidadi}

Katika lugha ya Kirombo kuna viambishi vinavyowakilisha nafsi na yambwa kwa idadi ya umoja na wingi ambapo: Nafsi ni kategoria ya kisarufi itumikayo kuonesha wahusika katika hali fulani maalum. Kuna nafsi kuu tatu; nafsi ya kwanza, nafsi ya pili na nafsi ya tatu, ambapo nafsi ya kwanza ni kategoria inayoonesha kwamba mzungumzaji anajirejea mwenyewe au anarejea kundi la watu ambalo yeye mwenyewe hujumuishwa ndani mwake. Katika Kirombo nafsi hii huwakilishwa na: nyáni au kiambishi patanishi ngi- katika umoja na katika wingi huwakilishwa na swáso na kiambishi patanishi du-.

Aidha, nafsi ya pili ni kategoria inayoonesha kwamba mzungumzaji anarejea kwenye mtu au kundi la watu anayesema nae ana kwa ana. Katika Kirombo nafsi hii huwakilishwa na fafe na kiambishi patanishi u- katika umoja na katika wingi huwakishwa na noano na kiambishi patanishi m-. Pia, nafsi ya tatu ni kategoria inayoonesha kwamba mtu au kitu kinachorejelewa hakina uhusiano wa ana kwa ana na msemaji au mzungumzaji. Katika Kirombo nafsi hii huwakilishwa na fé na kiambishi patanishi a- katika umoja na katika wingi huwakilishwa na fafo na kiambishi patanishi fa-.

Pamoja na hayo, yambwa ni kipashio cha sentensi au tungo ambacho huelekezwa tendo au lengo (Massamba, 2016). Katika Kirombo nafsi zote zinaweza kuambatana na yambwa katika tungo, ambapo yambwa katika nafsi ya kwanza huwakilishwa na kiambishi -m- katika umoja na fa- katika wingi. Aidha, katika nafsi ya pili yambwa huwakilishwa na kiambishi -m- katika umoja na m- katika wingi. Pia, katika nafsi ya tatu yambwa huwakilishwa na kiambishi -m- katika umoja na fa- katika wingi. 
Jedwali la 1: Viambishi vya Nafsi na Yambwa Kiidadi

\begin{tabular}{|c|c|c|c|c|c|}
\hline \multicolumn{2}{|c|}{ Nafsi } & \multirow{2}{*}{$\begin{array}{l}\text { Idadi } \\
\text { Umoja } \\
\text { ngi- }\end{array}$} & \multirow{2}{*}{$\frac{\text { Wingi }}{d u-}$} & \multirow{2}{*}{$\begin{array}{l}\text { Mifano } \\
\text { Ngilekaba } \\
\text { Dulekaba }\end{array}$} & \multirow{2}{*}{$\begin{array}{l}\text { Maana } \\
\text { Nilipiga } \\
\text { Tulipiga }\end{array}$} \\
\hline & Nafsi & & & & \\
\hline I & Yambwa & m- & fa- & $\begin{array}{l}\text { Ngilemkaba } \\
\text { Dulefakaba }\end{array}$ & $\begin{array}{l}\text { Nilimpiga } \\
\text { Tuliwapiga }\end{array}$ \\
\hline II & Nafsi & $\mathrm{u}-$ & m- & $\begin{array}{l}\text { Uleamba } \\
\text { Mleamba }\end{array}$ & $\begin{array}{l}\text { Ulisema } \\
\text { Mlisema }\end{array}$ \\
\hline & Yambwa & m- & m- & $\begin{array}{l}\text { Ulemmbia } \\
\text { Mlemmbia }\end{array}$ & $\begin{array}{l}\text { Ulimwambia } \\
\text { Mlimwambia }\end{array}$ \\
\hline & Nafsi & a- & fa - & $\begin{array}{l}\text { Alekunda } \\
\text { Falekunda }\end{array}$ & $\begin{array}{l}\text { Alipenda } \\
\text { Walipenda }\end{array}$ \\
\hline III & Yambwa & m- & fa- & $\begin{array}{l}\text { Alemkunda } \\
\text { Falefakunda }\end{array}$ & $\begin{array}{l}\text { Alimpenda } \\
\text { Waliwapenda }\end{array}$ \\
\hline
\end{tabular}

Chanzo: (uwandani).

Ukichunguza katika jedwali la 7 hapo juu utaona kuwa, kuna viambishi vya nafsi na mofimu za yambwa zilizowekwa katika hali ya umoja na wingi na mifano ya matumizi yake.

\section{UCHANGANUZI WA RUWAZA YA TONI KATIKA VITENZI VISOUKOMO CHANGAMANI.}

Katika uchanganuzi wa ruwaza ya toni katika vitenzi visoukomo changamani tumeanza na vile vyenye idadi ndogo ya silabi hadi vile vyenye silabi nyingi. Hivyo, sehemu hii tutaanza na vitenzi visoukomo changamani vya silabi mbili za shina hadi vile vya silabi sita na kumalizia na vitenzi visoukomo vya silabi moja ya shina kutokana na utofauti wake wa ujitokezaji wa ruwaza ya toni. Bila shaka hapa napo tuna maswali ya kujibu: swali ni kwamba, TJM kwenye vitenzi visoukomo changamani hujitokeza katika silabi ya ngapi ya shina? Ili kuweza kujibu maswali haya kwa uwazi tunapaswa kuchunguza data ya (4) hapa chini:
4. a) imúfésa
b) imúbóryómwa
c) imúdósíryáo
d) imúténgénésyáse
e) imúsóryómólyánáse

'kumfesa'

'kumbomoa'

'kumpandishia hapo'

'kuwatengenezea tena'

'kuwachomolea tena'
( CJJC)

(CJJJC)

(CJJJJC)

(CJJJJJC)

(CJJJJJJC)
Ukitazama data (4) hapo juu kwa makini, unaona tofauti za wazi kwenye hiyo mifano ya vitenzi visoukomo zikijitokeza. Tunaona kwamba mifano ya vitenzi vilivyopo hutofautiana katika idadi ya silabi na idadi ya tonijuu zinazojitokeza katika makundi hayo ya vitenzi. Kutokana na tofauti hizo, hatuna budi kuchunguza kiimbotoni msingi katika lugha ya Kirombo kwa kuchunguza kila kitenzi katika data hii kuanzia mwanzo hadi mwisho. Hivyo, tunaweza kupitia kila silabi katika kila kitenzi kisoukomo changamani kuona ni toni gani inatangulia, ipi inaifuatia na ipi hujitokeza mwishoni mwa shina la vitenzi hivyo katika lugha hii. Katika mifano ya data (4) inaonekana kuwa, kila kitenzi kinaanza na tonichini na kufuatiwa na tonijuu inayoenda hadi silabi ya mwisho kasoro moja na silabi ya mwisho ina tonichini. Hivyo, tunaona ujitokezaji wa kiimbotoni msingi katika (4) (a - e) ni CJJC, CJJJC, CJJJJC, CJJJJJC na CJJJJJJC. Kwa hiyo, tunaweza kuhitimisha kuwa, kiimbotoni msingi cha toni katika vitenzi vya lugha ya Kirombo ni CJC.

Pamoja na hayo, tulieleza tutatumia mkabala wa Tonijuu Msingi katika uibuzi wa ruwaza ya toni kutoka umbo la ndani kwenda umbo la nje la vitenzi visoukomo changamani vya Kirombo. Katika kufanya hivyo tunapaswa kujua, tonijuu msingi (kuanzia sasa TJM) tuliyoibaini katika kiimbotoni msingi inahusishwa na silabi ipi katika kitenzi kisoukomo changamani? Ama kwa hakika hapa 
napo hakuna jibu la mkato. Hii hutokana na ukweli kwamba, kila lugha ina taratibu zake maalumu za upachikaji wa TJM katika mashina ya vitenzi au maneno mengine.

Kabla ya kuendelea na mjadala wa kutaka kujua TJM huhusishwa na silabi ipi katika lugha ya Kirombo, yafaa tufafanue hapa lau kwa kina kwanza tunaposema TJM tuna maanisha nini ndipo tuendelee na mjadala wetu. Lugha nyingi za Kibantu katika miaka ya 500 baada ya Kristu zilionekana kuwa na toni mbili tofauti ambazo ni tonijuu (J) na tonichini (C) (Meeussen, 1980; Coupez, 1983; Marlon na Odden, 2014 na Hyman, 2017). Utofauti wa toni hizo umetokana na kuwa na viwango tofauti kimsikiko. Yaani, Tonijuu (kuanzia sasa J) ni toni ambayo silabi inayohusishwa nayo inapotamkwa huwa ina msikiko unaoelekea juu, wakati tonichini (kuanzia sasa C) ni toni ambayo silabi inayohusishwa nayo inapotamkwa msikiko unaelekea chini (Massamba 2011: 171, Möller, 2014:12). Toni J hualamishwa kwa alama ['] na toni $C$ hutumia alama [' ].

Hata hivyo, wapo wataalamu wanaoona kuwa awali lugha za Kibantu zilikuwa na toni $\mathrm{J}$ moja na toni kapa (Ø) (toni J Vs Ø) (Stevick, 1969; Nash, 1992). Wataalamu hawa hudai kuwa toni kapa kimsikiko ni hafifu sana na hivyo kuona kuwa haipo. Madai ya kundi hili kwa hakika hayasigani na madai ya kundi la kwanza kwa sababu wote wanatambua kuwa awali lugha za Kibantu zilikuwa na viwango

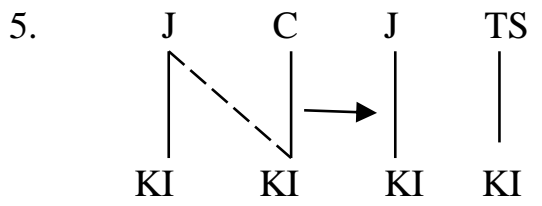

Violezo vya 5 na 6 hapo juu vinaonesha kuwa toni $\mathrm{J}$ inasambaa kuelekea kulia mwa shina la neno na kuungana na toni $\mathrm{C}$ ya silabi inayofuatia katika shina na matokeo yake ni toni TS. Aidha, kiolezo cha 6 kinaonesha kuwa toni $\mathrm{C}$ inasambaa kuelekea kulia mwa shina na kuungana na toni $\mathbf{J}$ ya silabi inayofuatia na matokeo yake ni uzalishaji wa toni TP.

Katika lugha ya Kirombo kuna toni tatu kati ya hizo katika vitenzi visoukomo ni toni $\mathrm{J}$, toni $\mathrm{C}$ na toni TS. Chunguza mifano ifuatayo katika 7 hapa chini: viwili tofauti vya toni na ambavyo vilitofautishwa kulingana na msikiko kiutamkaji.

Kulingana na mabadiliko ya kiisimu na michakato ya kifonolojia na kimofolojia katika lugha imesababisha viwango hivyo vya toni kuathiriwa na kuanza kuonekana kuwa na mabadiliko ya kitoni ya Ushukaji wa toni $\mathrm{J}$ kuwa na toni kontua, na kwa kiasi kidogo sana ushukaji wa toni $C$.

Ila inapotokea toni $\mathrm{J}$ na toni $\mathrm{C}$ zimeungana kutokana na michakato ya kifonolojia na kimofolojia katika lugha, matokeo yake yanaweza kuwa ama upatikanaji wa Tonipanda (TP) au Tonishuka (TS) ikiwa na maana kuwa, toni TP ni toni ambayo silabi inayohusishwa nayo hutamkwa kwa msikiko unaoanzia chini kwenda juu. Hivyo, toni TP ni matokeo ya muunganiko wa toni $\mathrm{C}$ na toni J. Aidha, toni TS ni toni ambayo silabi inayohusishwa nayo inapotamkwa huwa na msikiko ambao uanzia juu kushuka chini. Toni TS ni matokeo ya muunganiko wa toni $\mathrm{J}$ na toni $\mathrm{C}$. Ongezeko la aina hizi mbili za toni (TP na TS) ndio unaofanya idadi ya toni katika lugha nyingi za Kibantu kuwa nne. TP na TS zinaweza kutokea si tu kwa kuungana kama ilivyooneshwa bali pia kwa msambao wa toni $\mathrm{J}$ na toni $\mathrm{C}$ kutoka kushoto kuelekea kulia mwa neno (Snider, 1999:57 na Hyman, 2017:2). Chunguza kiolezo cha msambao wa toni $\mathrm{J}$ na toni $\mathrm{C}$ kifuatacho katika 87 ukilinganisha na 88 hapa chini.

6.

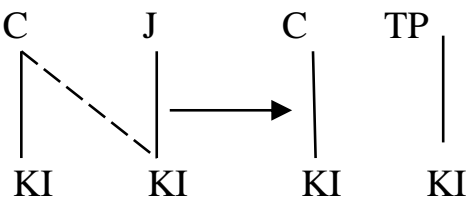
7. a) iséka
CJC
'kucheka'
b) itéréfa
CJJC
c) itôla
CSC
d) ifîna
CSC
'kuomba'
'kudonoa'
'kuimba'

Katika data 7 hapo juu ina mifano ya vitenzi vyenye toni tatu tofauti ambazo ni toni $\mathrm{J}$, toni $\mathrm{C}$ na toni TS. Yafaa ieleweke kuwa silabi zisizoalamishwa zina toni C. Hivyo, katika 7(a-b) kuna toni J na toni C pekee katika mifano hiyo ya vitenzi, wakati katika $7(\mathrm{c}-\mathrm{d})$ kuna toni $\mathrm{C}$ na toni TS ambayo ni matokeo ya muunganiko wa toni $\mathrm{J}$ na toni $\mathrm{C}$. 
Kwa kuwa imekwisha elezwa kwamba toni $\mathrm{J}$ na toni $\mathrm{C}$ ndizo toni pekee zilizokuwepo awali katika lugha nyingi za Kibantu kabla ya utokeaji wa mabadiliko ya Kiisimu katika lugha hizo, na ambazo zikiungana matokeo yake ni uzalishaji wa ama toni TP au toni $\mathrm{TS}$, basi toni $\mathrm{J}$ na toni $\mathrm{C}$, zote uchukuliwa kuwa ni Toni Msingi katika lugha za Kibantu. Kwa kuwa toni J msingi imebainika kuwa na mvumo wa juu kimsikiko kuliko toni $\mathrm{C}$, na kwa kuwa baadhi ya wataalamu wanaiona toni $\mathrm{C}$ kama toni kapa $(\varnothing)$ na ambayo mara nyingi haialamishwi inapotokea na toni $\mathrm{J}$ katika neno moja, basi tunachukulia kifonolojia toni $\mathrm{J}$ ndiyo msingi wa toni zote katika lugha.

Kutokana na maelezo ya kinadharia yaliyotolewa hapo juu, uchanganuzi wa uibuzi wa toni katika umbo la nje kutoka umbo la ndani katika Kirombo utatumia toni $\mathrm{J}$ kama toni msingi (TJM). Kwa mujibu wa Möller (2014), Toni Msingi huweza kujidhihirisha kwa namna tofauti kwenye maneno mbalimbali. Kwa kawaida maneno hubeba sauti na toni msingi inayohusishwa na silabi maalumu. Pia, neno linaweza kusitiri toni msingi bila kuhihusisha na silabi. Ikitokea hivyo, neno husika litakuwa na toni inayoelea. Aidha, lugha inaweza kuwa na maneno yasiyo na toni msingi na hivyo kufanya maneno hayo kuitwa maneno yasiyo na tone. Ni mara chache sana kukuta lugha yenye kusitiri aina zote za maneno yenye aina hizo za toni msingi zilizotajwa (Snider, 1999:6). Tuchunguze kiolezo kifuatacho katika 8 hapa chini:

\section{8. a) Neno lenye toni na silabi \\ b) Neno lenye toni inayoelea \\ c) Neno lisilo na toni}

$\mathrm{T}$

\#\$

\#\$

Alama (T) imetumika kama toni, alama (\$) imetumika kama silabi na alama (\#) imetumika kuonesha mwanzo na mwisho wa neno. Ukichunguza violezo vilivyopo katika 8 hapo juu utabaini kwamba, kiolezo cha 8(a) kina toni msingi iliyohusishwa na silabi maalumu kwenye neno, wakati kiolezo cha 8(b) kinaonesha kuwa kina toni msingi inayoelea kwa sababu haijahusishwa na silabi maalumu katika neno na mwisho kiolezo cha 8(c) kinaonesha neno ambalo halina toni msingi na hivyo kuwa neno lisilo na toni.

Katika lugha ya Kirombo toni msingi hujitokeza kwa namna ya kwanza iliyotajwa na Möller (keshatajwa) ambapo TJM huhusishwa na silabi maalumu kwenye kitenzi katika umbo la ndani, kwa kufuata utaratibu unaofanana kwa vitenzi visoukomo vyote vya lugha inayohusika. Ili tuweze kujua ni silabi ipi katika kitenzi kisoukomo changamani huhusishwa na TJM katika Kirombo, hatuna budi kufanya uchanganuzi wa kimajaribio utakaoongozwa kwa kanuni maalumu. Tunapendekeza kanuni itakayotumika katika upachiaji wa toni $\mathbf{J}$ msingi kwenye silabi maalumu ya shina la kitenzi kisoukomo kuwa ni kanuni ya jumla ya upachikaji wa toni J msingi katika silabi maalumu ya shina la kitenzi kisoukomo changamani. Kanuni hii iundwe baada ya kufanyika kwa majaribio anuai ambayo ndiyo yanayotupatia idhibati kuwa TJM hupachikwa kwenye silabi ipi.

Katika kufanya hivyo, tumechukua kitenzi kisoukomo changamani cha silabi mbili za shina katika lugha ya Kirombo 'imú tésa' 'kumutesa' na kulifanyia majaribio mbalimbali ya kiukokotozi hadi tulipobaini kuwa, vitenzi visoukomo changamani vya Kirombo vina TJM mbili ambapo moja hujitokeza katika mofimu ya yambwa na ya pili katika silabi ya kwanza ya shina.

Kama tulivyokwishasema hapo juu, ni wazi kwamba mofimu inayowakilisha yambwa kwenye Kirombo katika kitenzi 'imú tésa' imebeba toni J. Ukichunguza mahali pa utokeaji utagundua kuwa imefuatia KA na kuitangulia silabi ya kwanza ya shina ambayo nayo ina toni J. Kwa kuwa zote (yambwa na silabi ya kwanza ya shina) zina toni $\mathbf{J}$ na kwa kuwa yambwa imeitangulia silabi ya shina, basi ichukuliwe kuwa zote zinapaswa zihusishwe na TJM kama inavyoonekana hapa chini: 
imú tésa

/i mu te sa/

9. $\quad$ i mu te sa<smiles>I[14IH]I</smiles>

[i mú té sa] 'kumutesa'

'Umbo la ndani'

'Upachikaji wa TJM'

'Uhusishaji wa Tonichini na Vitamkwa'

'Umbo la nje'
Matokeo ya ukokotozi wa (9) hapo juu, yanakubalika katika lugha ya Kirombo [i mú te sa]. Matokeo haya yanaonesha kuwa, ruwaza ya ujitokeza wa toni katika kitenzi kisoukomo changamani [i mú té sa], huanza na toni $\mathrm{C}$ katika kiambishi kisoukomo /i-/, ikifuatiwa na toni $\mathrm{J}$ katika mofimu ya yambwa, kisha kufuatiwa na toni J katika silabi ya kwanza ya shina na kuishia na toni C katika silabi ya mwisho ya shina. Hata hivyo, pamoja na ukubalifu wa matokeo ya 9 hapo juu, njia ya ukokotozi huu inaweza isitufae sana katika uibuzi wa toni zinazojitokeza kwenye data zenye silabi nyingi za shina na ambazo huitajia utekelezwaji wa kanuni nyingine kama vile msambao wa toni $\mathrm{J}$ kuelekea kulia na sharti la ukubalifu ambalo halihitajii mistari ya uhusihano kukingamana hasa toni inaposambaa. Hii ni kwa sababu haitafahamika kwa urahisi kuwa toni $\mathbf{J}$ inayosambaa ni ipi kati ya silabi ya kwanza ya shina na ile ya mofimu ya yambwa.

Pia, utoufaafu wa njia ya ukokotozi wa 9, umetokana na ukweli kwamba, toni $\mathbf{J}$ ya yambwa na ile ya shina zote zimejitokeza kama TJM na ambazo hufuatana. Katika Kirombo, toni J mbili zinapofuatana hususani toni $\mathbf{J}$ ya yambwa na ya shina, ile ya yambwa huwa na nguvu zaidi ya toni $\mathrm{J}$ ya shina, yaani, kimatamshi toni J ya mofimu ya yambwa inapotamkwa huwa na mvumo wa juu kimsikiko kuliko toni $\mathbf{J}$ ya silabi ya kwanza ya shina. Maelezo hayo yanashadidiwa na udondoaji ufuatao:

“........utamkaji wa toni katika mfululizo wa tonijuu huzidi kufifia kadri unavyozidi kusonga mbele. Lugha yenye ufifishaji wa tonijuu inaweza kuwa na sheria kama vile; tonijuu inayotanguliwa na tonijuu nyingine huwa ya kidatu cha chini zaidi kuliko ile tonijuu iliyoitangulia au tonijuu inayoifuatia tonijuu nyingine ambayo imeathiriwa na ushukaji toni kihatua, huwa na thamani ya kifonetiki sawa na tonijuu iliyoitangulia' '(Massamba, 2011:172).

Kutokana na dondoo hili, katika Kirombo toni J ya silabi ya kwanza ya shina huwa hafifu inapotanguliwa na toni $\mathrm{J}$ ya mofimu ya yambwa, ukilinganisha na zile toni $\mathbf{J}$ za shina katika silabi ya kwanza kwenye vitenzi visoukomo sahili. Kulingana na sababu hii tunapendekeza TJM ya shina ambayo ni dhaifu idondoshwe ili kuruhusu kanuni ya msambao wa toni $\mathrm{J}$ kuelekea kulia na sharti la ukubalifu liweze kutekelezwa pasi mistari kukingamana. Hii ni kwa sababu tunachukulia kwamba toni $\mathbf{J}$ inayosambaa ni ile ya mofimu ya yambwa ambayo ndiyo yenye nguvu kuliko ile ya silabi ya kwanza ya shina. Hivyo, katika kushughulikia suala la udondoshaji wa toni TJM ya silabi ya kwanza ya shina, tunapendekeza kuunda kanuni ya udondoshaji wa TJM ya shina kama inavyoonekana hapa chini:

\section{Kanuni ya 1: Udondoshaji wa TJM ya Shina}

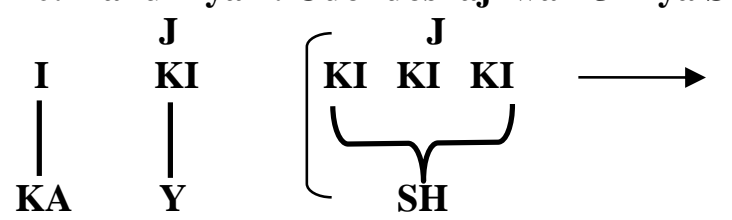

Kanuni ya 1 inasomeka kuwa, TJM ya silabi ya kwanza ya shina idondoshwe iwapo na iwapo tu

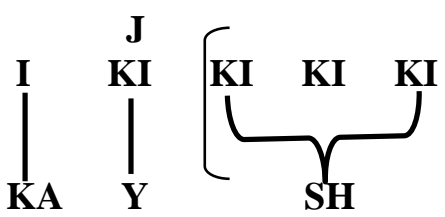

imetanguliwa na TJM ya yambwa. Sharti kubwa la kanuni hii ni kwamba, huwezi kudondosha TJM 
kabla hujahihusisha na silabi ya kwanza ya shina la kitenzi husika. Ikumbukwe kuwa, udondoshaji wa TJM ya shina umefanyika kwa sababu za msingi zilizokwisha fafanuliwa hapo juu ya kwamba husaidia kuruhusu kanuni nyingine kufanya kazi hususani kanuni ya msambao wa toni $\mathrm{J}$ kuelekea kulia mwa shina la kitenzi.

$$
\begin{aligned}
& \text { imú tésa } \\
& \text { /i mu te sa/ }
\end{aligned}
$$

11.<smiles>C1COCCO1</smiles><smiles>I[14CH][13IH]I</smiles><smiles></smiles><smiles>CCCCCCCC</smiles><smiles>C[13CH][13CH]I</smiles>
[imú tésa]
Baada ya kuunda kanuni hiyo, tunaweza sasa kuchunguza mifano ya ukokotozi itakayotumia kanuni hizo anuwai hatua kwa hatua katika vitenzi visoukomo changamani vilivyopo katika data (11) kama ifuatavyo:

'Udondoshaji wa TJM ya silabi ya 1 ya shina'

'Upachikaji wa TJM'

'Usambaaji wa TJM'

'Uhusishaji wa Tonichini na Vitamkwa'

'Sharti la Ukubalifu'

'Umbo la nje'

Ukokotozi wa (11) hapo juu, umetupatia matokeo yanayokubalika katika Kirombo [imú tésa]. Matokeo ya ukokotozi wa (11) yanaonesha kwamba, ruwaza ya ujitokezaji wa toni katika kitenzi kisoukomo changamani [imú tésa], huanza kwa toni C katika kiambishi kisoukomo /i-/, ikifuatiwa na toni $\mathrm{J}$ ya mofimu ya yambwa ambayo nayo usambaa hadi silabi ya kwanza ya shina na kuishia na toni $\mathrm{C}$ katika silabi ya mwisho ya shina. Aidha, ukichunguza kwa makini ukokotozi (11) utagundua kuwa, kanuni ya udondoshaji wa TJM ya silabi ya kwanza ya shina imetekelezwa baada ya utekelezwaji wa kanuni ya jumla ya upachikaji wa TJM katika mofimu ya yambwa na silabi ya kwanza ya shina la kitenzi husika. Aidha, kanuni ya udondoshaji wa TJM ya shina katika ukokotozi (11) imeruhusu ufanyaji kazi wa kanuni ya msambao wa TJM kuelekea kulia kabla ya utekelezwaji wa hatua ya uhusishaji wa Tonichini na Vitamkwa. Pia, katika ukokotozi wa uibuzi wa toni katika (11), Sharti la ukubalifu limetekelezwa katika hatua ya mwisho ya ukokotozi.

Matokeo ya (11) hapo juu, yametuwekea msingi wa uchanganuzi kwa kuchukulia kwamba, katika Kirombo, vitenzi visoukomo changamani vina TJM mbili: ile ya mofimu ya yambwa na ya silabi ya kwanza ya shina. Ila, TJM ya silabi ya kwanza ya shina ni hafifu na hivyo udondoshwa ili kupisha kanuni ya msambao wa toni J kufanya kazi. Kwa kutumia msingi huu, basi tunaweza kuendelea na uchanganuzi wa vitenzi visoukomo changamani katika (4.1) kama ifuatavyo: 


\section{Ujitokezaji wa Ruwaza ya Toni katika Vitenzi Visoukomo Changamani vya Silabi mbili za Shina}

Kutokana na matokeo ya utafiti, imegundulika kuwa vitenzi visoukomo changamani vya silabi mbili za shina vina ruwaza ya ujitokezaji wa toni wa CJC. Chunguza data (12) hapa chini:

$\begin{array}{ll}\text { 12. a) i múfé sa } & \text { 'kumambia' } \\ \text { b) i múbá sha } & \text { 'kumchonga' } \\ \text { c) i mú kó rya } & \text { 'kumpika' } \\ \text { d) i múfé ya } & \text { 'kumkatia', } \\ \text { e) i múbá rya } & \text { 'kumpasua' } \\ \text { f) i múté wa } & \text { 'kumchagua' } \\ \text { g) i mú ló swa } & \text { 'kumota ndoto' } \\ \text { h) i músú ma } & \text { 'kumchimba' }\end{array}$

Ukichunguza kwa makini data ya (12) utagundua kuwa, vitenzi vyote visoukomo changamani vina silabi mbili za shina, mofimu /-m-/ ya yambwa na kiambishi kisoukomo /i-/. Pia, utagundua kuwa vitenzi vyote vimeanza na toni $\mathrm{C}$, ikifuatiwa na toni j na kuishia na toni C. Hivyo, kuwa na ruwaza ya ujitokezaji wa toni wa CJC. Hii ikiwa na maana kwamba, kiambishi awali / i- / ambacho ni kisoukomo cha vitenzi visoukomo vya Kirombo kina toni $\mathrm{C}$, ambayo hufuatiwa na toni $\mathrm{J}$ ya silabi ya kwanza ya shina na silabi zote za mwisho wa shina la kitenzi kisoukomo changamani zimebeba toni $\mathrm{C}$. Ukokotozi wa uibuzi wa toni katika umbo la nje kutoka umbo la ndani umefanyika kwa kuzingatia kanuni ya jumla ya upachikaji wa TJM katika silabi ya kwanza ya shina na mofimu ya yambwa. Hebu tuchunguze mfano wa ukokotozi katika 13 hapa chini:

$\begin{array}{ll}\text { i múfé sa } & \text { 'kumambia' } \\ \text { /i mu fe sa/ } & \text { 'Umbo la ndani' }\end{array}$

13. i mu fe sa<smiles>I[14CH]I</smiles>

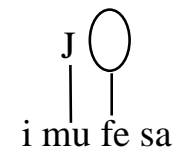

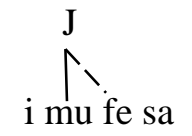<smiles>C[AlH]C(I)[AlH]I</smiles><smiles>C[13IH]I</smiles>
[i múfé sa]
'Upachikaji wa TJM'

'Udondoshaji wa TJM ya shina'

'Msambao wa toni J'

'Uhusishaji wa Tonichini na Vitamkwa'

'Sharti la Ukubalifu'

'Umbo la nje'
Katika ukokotozi wa (13) hapo juu, tumepata matokeo yanayokubalika katika usemaji wa Kirombo [i múfé sa]. Matokeo ya ukokotozi wa (13) yanaonesha kwamba, ruwaza ya ujitokezaji wa toni katika kitenzi kisoukomo changamani [i múfé sa], huanza kwa toni C, katika kiambishi kisoukomo /i-/, ikifuatiwa na toni J katika mofimu ya yambwa, kisha kufuatiwa na toni $\mathrm{J}$ katika silabi ya kwanza ya shina na kuishia na toni $\mathrm{C}$ katika silabi ya mwisho ya shina. Aidha, ukichunguza kwa makini ukokotozi wa (13), utagundua pia kwamba, kanuni ya upachikaji wa TJM katika silabi ya kwanza ya shina na katika mofimu ya yambwa imetekelezwa kabla ya utekelezwaji wa kanuni ya udondoshaji wa TJM ya shina kama inavyodaiwa katika sheria za Nadharia ya FVH kwa lengo la kuruhusu kanuni ya msambao kufanya kazi. Pia, kanuni ya msambao wa toni $\mathrm{J}$ kuelekea kulia mwa 
shina imetekelezwa kabla ya utekelezwaji wa hatua ya uhusishaji wa tonichini na vitamkwa na mwisho katika ukokotozi wa (13), limetekelezwa Sharti la Ukubalifu. Tuongeze mfano mmoja wa ukokotozi katika (14) kabla ya kuendelea na uchanganuzi wa vitenzi visoukomo changamani vya silabi tatu za shina. $\begin{array}{ll}\text { i mú bá sha } & \text { 'kumchonga' } \\ \text { /i mu ba sha/ } & \text { 'Umbo la ndani' }\end{array}$

$\begin{array}{ll}\text { J } & \text { J }\end{array}$

14. i mu ba sha

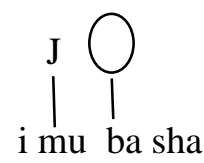

'Udondoshaji wa TJM ya shina'

'Msambao wa toni J'

'Uhusishaji wa Tonichini na Vitamkwa'

'Sharti la Ukubalifu'

'Umbo la nje'
Katika ukokotozi (14) hapo juu, tumepata matokeo yanayokubalika katika usemaji wa Kirombo [i mú bá sha]. Matokeo ya ukokotozi (14) yanaonesha kwamba, ruwaza ya ujitokezaji wa toni katika kitenzi kisoukomo changamani, huanza kwa toni $\mathrm{C}$, katika kiambishi kisoukomo /i-/, ikifuatiwa na toni J katika mofimu ya yambwa, kisha kufuatiwa na toni $\mathrm{J}$ katika silabi ya kwanza ya shina na kuishia na toni $\mathrm{C}$ katika silabi ya mwisho ya shina. Aidha, ukichunguza kwa makini ukokotozi wa (14), utagundua pia kwamba, kanuni ya upachikaji wa TJM katika silabi ya kwanza ya shina na katika mofimu ya yambwa imetekelezwa kabla ya utekelezwaji wa kanuni ya udondoshaji wa TJM ya shina kama inavyodaiwa katika sheria za Nadharia ya FVH kwa lengo la kuruhusu kanuni ya msambao kufanya kazi. Pia, kanuni ya msambao wa toni J kuelekea kulia mwa shina imetekelezwa kabla ya utekelezwaji wa hatua ya uhusishaji wa tonichini na vitamkwa na mwisho katika ukokotozi wa (14), limetekelezwa Sharti la Ukubalifu. Kulingana na matokeo ya ukokotozi wa (13) na (14) hapo juu, yote yanakubalika katika usemaji wa Kirombo na hivyo, hatuna budi kuendelea na uchanganuzi wa vitenzi visoukomo changamani vya silabi tatu za shina katika (4.2) hapa chini:

\section{Ujitokezaji wa Ruwaza ya Toni katika Vitenzi Visoukomo Changamani vya Silabi Tatu za Shina}

Baada ya kuona ruwaza ya ujitokezaji wa toni katika vitenzi visoukomo changamani vya silabi mbili za shina, sasa tuendelea na uchanganuzi wa uibuzi wa toni katika vitenzi visoukomo changamani vya silabi tatu za shina la kitenzi. Katika Kirombo, ujitokezaji wa ruwaza za toni katika aina hii ya vitenzi vya silabi tatu ni wa CJJJC. Tuchunguze data (15) hapa chini:
15. a) imútéréfa
'kumomba'
b) imúbóryómwa
'kumbomoa'
c) imúkámáta
'kumpeleka'
d) imúkódírya
'kumvutia'
e) imúsémbérya
'kumsingizia'
f) imúsánírya 
g) imútúkúlya 'kumangalia'

Ukichunguza kwa makini data $15(\mathrm{a}-\mathrm{g})$ hapo juu, utagundua kwamba, vitenzi visoukomo changamani vilivyopo vina idadi sawa ya silabi tatu za shina la kitenzi na mofimu /-m-/ ya yambwa. Pia, vyote vimeanza kwa kiambishi kisoukomo /i-/ na ambacho ni kiambishi awali cha vitenzi visoukomo vya Kirombo. Pia, kitoni, utagundua kwamba, vitenzi visoukomo changamani vyote katika (15) vina toni $\mathbf{J}$ tatu ambazo hujitokeza katika mofimu ya yambwa na kusambaa hadi silabi ya pili ya shina la vitenzi husika na kiambishi kisoukomo na silabi ya mwisho ya shina vyote vimebeba toni C. Hivyo, kutokana na data (15) utagundua kwamba, ruwaza ya ujitokezaji wa toni katika vitenzi hivyo ni CJJJC. Yaani, vitenzi vyote vimeanza na toni $\mathrm{C}$ katika kiambishi kisoukomo, ikifuatiwa na toni J katika mofimu ya yambwa, kisha kufuatiwa na toni $\mathbf{J}$ katika silabi ya kwanza na ya pili ya shina na kuishia na toni $\mathrm{C}$ katika silabi ya mwisho ya shina. katika ukokotozi wa uibuzi wa toni katika umbo la nje kutoka umbo la ndani la vitenzi visoukomo changamani vya silabi tatu za shina, kanuni ya msambao wa toni J kuelekea kulia, kanuni ya 1 ya udondoshaji wa TJM ya shina, pamoja na kanuni nyingine za uchanganuzi zitazingatiwa. Chunguza ukokotozi wa uibuzi wa toni katika 16 hapa chini:
i mú bó ryó mwa 'kumbomoa'
/i mu bo ryo mwa/ 'Umbo la ndani'

16.

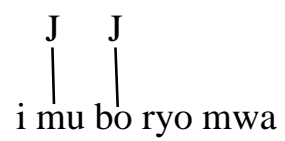

'Upachikaji wa TJM'<smiles>O[PbH](O)I</smiles>

'Udondoshaji wa TJM ya shina'

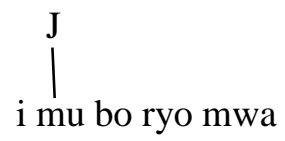

'Upachikaji wa TJM'<smiles>COC1CC1(C)[18OH]</smiles>
'Usambaaji wa TJM'<smiles>CCC(C)C</smiles>
i mu bo ryo mwa 'Uhusishaji wa Tonichini na Vitamkwa'<smiles></smiles>

[i mú bó ryó mwa]
'Sharti la Ukubalifu'

'Umbo la nje'
Ukokotozi wa data (16) hapo juu, umetupatia matokeo yanayokubalika katika usemaji wa Kirombo [i mú bó ryó mwa]. Matokeo ya ukokotozi (16) yanaonesha kwamba, ruwaza ya ujitokezaji wa toni katika kitenzi kisoukomo [i mú bó ryó mwa], huanza kwa toni C katika kiambishi kisoukomo /i-/ ambacho pia ni kiambishi awali cha vitenzi visoukomo vya Kirombo, ikifuatiwa na toni J katika mofimu ya yambwa, ambayo pia hufuatiwa na toni
J katika silabi ya kwanza na ya pili ya shina na kuishia na toni $\mathrm{C}$ katika silabi ya mwisho ya shina. Aidha, katika ukokotozi (16), kanuni za uchanganuzi zimezingatiwa hatua kwa hatua katika kupata matokeo yanayokubalika. Hii ina maana kuwa, kanuni ya jumla ya upachikaji wa TJM katika silabi ya kwanza ya shina na katika mofimu ya yambwa imetekelezwa katika hatua ya mwanzo ya ukokotozi. Pia, kanuni ya udondoshaji wa TJM 
ya shina imetekelezwa kabla ya utekelezwaji wa kanuni ya msambao wa toni J kuelekea kulia mwa shina, kisha kufuatiwa na hatua ya uhusishaji wa Tonichini na vitamkwa na kuishia na Sharti la Ukubalifu mwishoni mwa ukokotozi wa kitenzi kisoukomo changamani [i mú bó ryó mwa]. Tuongeze mfano mmoja wa ukokotozi katika 17 kabla ya kuendelea na uchanganuzi wa uibuzi wa ruwaza ya toni katika vitenzi visoukomo changamani vya silabi nne za shina. i mú sé mbé rya

/i mu se mbe rya/

17.<smiles>I[13CH]C(I)I</smiles><smiles>O=C(I)C(O)O</smiles>

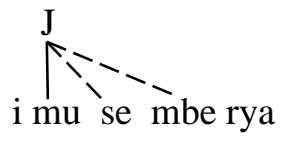

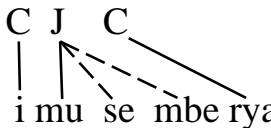<smiles>I[13CH][13CH](I)[13CH]I</smiles>

[i mú sé mbé rya]

\section{'Kumsingizia'}

'Umbo la ndani'

'Upachikaji wa TJM'

'Udondoshaji wa TJM ya shina'

'Msambao wa toni J'

'Uhusishaji wa Tonichini na Vitamkwa'
Katika ukokotozi wa (17) hapo juu, tumepata matokeo yanayokubalika katika usemaji wa Kirombo [i mú sé mbé rya]. Matokeo ya ukokotozi (17) yanaonesha kwamba, ruwaza ya ujitokezaji wa toni katika kitenzi kisoukomo [i mú sé mbé rya], huanza kwa toni C katika kiambishi kisoukomo /i-/ ambacho pia ni kiambishi awali cha vitenzi visoukomo vya Kirombo, ikifuatiwa na toni J katika mofimu ya yambwa, ambayo pia hufuatiwa na toni J katika silabi ya kwanza nay a pili ya shina na kuishia na toni $\mathrm{C}$ katika silabi ya mwisho ya shina. Aidha, katika ukokotozi (17), kanuni za uchanganuzi zimezingatiwa hatua kwa hatua katika kupata matokeo yanayokubalika. Hii ina maana kuwa, kanuni ya jumla ya upachikaji wa TJM katika silabi ya kwanza ya shina na katika mofimu ya yambwa imetekelezwa katika hatua ya mwanzo ya ukokotozi. Pia, kanuni ya udondoshaji wa TJM ya shina imetekelezwa kabla ya utekelezwaji wa kanuni ya msambao wa toni J kuelekea kulia mwa shina, kisha kufuatiwa na hatua ya uhusishaji wa
Tonichini na vitamkwa na kuishia na Sharti la Ukubalifu mwishoni mwa ukokotozi wa kitenzi kisoukomo changamani [ $i$ mú sé mbé rya]. Kulingana na matokeo ya ukokotozi wa uibuzi wa toni katika (16 na 17) kukubalika katika usemaji wa Kirombo, basi, tuendelee na uchanganuzi wa ruwaza ya vitenzi visoukomo changamani vya silabi nne za shina katika uibuzi wa toni katika umbo la nje kutoka umbo la ndani kama ifuatavyo:

\section{Ujitokezaji wa Ruwaza ya Toni katika Vitenzi Visoukomo Changamani vya Silabi Nne za Shina}

Katika 4.2 tumejadili kwa kina na tumeona ruwaza ya ujitokezaji wa toni katika vitenzi visoukomo changamani vya silabi tatu za shina. Aidha, tumeona namna kanuni ya udondoshaji wa TJM ya shina inavyoruhusu kanuni ya msambao wa toni $\mathbf{J}$ kuelekea kulia mwa shina la kitenzi kufanya kazi katika ukokotoaji wa hivyo vitenzi visoukomo 
changamani vya silabi tatu za shina. Katika sehemu hii tumeendelea na uchanganuzi wa ruwaza ya ujitokezaji wa toni katika vitenzi visoukomo changamani vya silabi nne za shina katika kuibua toni kwenye umbo la nje kutoka umbo la ndani la vitenzi husika. Utafiti umebaini kuwa, ujitokezaji wa ruwaza za toni katika vitenzi visoukomo changamani vya silabi nne za shina zipo katika makundi mawili ambayo ni CJJJC na CJJCC.

Tuanze kuchunguza ruwaza ya ujitokezaji wa toni katika kundi la kwanza la vitenzi visoukomo changamani vya silabi nne za shina lenye CJJJC. Tuchunguze data (18) hapa ifuatayo:
18. a) imúbéryéngésya
'kumuumiza'
b) imúlémbéryílya
'kumsingizia'
c) imúdólyíryílya
'kumpandia'
d) imúséngéryílya
'kumsogelea'
e) imútámbúkyírya
'kumtembelea'

Ukichunguza data (18) kwa makini utagundua kwamba, vitenzi visoukomo changamani vilivyopo katika data (18) vina idadi sawa ya silabi za shina ambazo ni nne na zina muundo wenye mfuatano unaofanana. Pia, utagundua kwamba, vitenzi visoukomo changamani vyote katika (18) vimeanza kwa kiambishi kisoukomo / i-/ na ambacho ni kiambishi awali cha vitenzi visoukomo vya Kirombo. Sanjari na hayo, utagundua pia, vitenzi visoukomo changamani viliyopo katika 18(a-e), shina lake limeishilia yenye kiyeyusho [ j ]. Kitoni, vitenzi vya data (18) hapo juu, vina toni $\mathbf{J}$ tatu za shina na toni J moja ya mofimu ya yambwa /m-/ na vitenzi hivyo vina toni $\mathrm{C}$ katika kiambishi kisoukomo /i-/ na katika silabi ya mwisho ya shina pia vina toni C. ujitokezaji wa toni katika data (18) ndio unaofanya kundi hili la vitenzi visoukomo changamani vya silabi nne za shina viwe na ruwaza ya CJJJJC. Yaani, kiambishi kisoukomo /i-/ kina toni $\mathrm{C}$, inayofuatiwa na toni $\mathrm{J}$ katika mofimu ya yambwa na ambayo usambaa kuelekea kulia mwa shina hadi silabi ya mwisho kasoro moja na kuishia na toni $\mathrm{C}$ katika silabi ya mwisho ya shina. Kwa kuwa msambao wa toni J katika kundi hili la vitenzi visoukomo changamani hausigani na ule wa vitenzi visoukomo changamani wa silabi tatu za shina, basi, kanuni za ukokotozi zilizotumika ndizo zilizotumika pia kukokotolea kundi hili la silabi nne za shina kama inavyoonekana hapa chini: imúbéryéngésya

/i mu be rye nge sya/

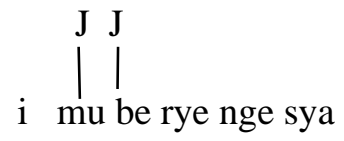

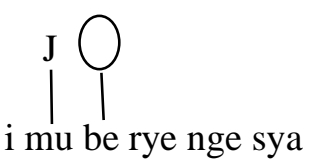<smiles>CC=C(C)C</smiles>
i mu be rỳe nge sya<smiles>CC(C)(I)C1CCCCC1</smiles>

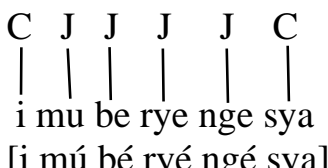

Ukokotozi wa (19) hapo juu, umetupatia matokeo yanayokubalika katika usemaji wa Kirombo [i mú

\section{'Kumuumiza'}

'Umbo la ndani'

'Upachikaji wa TJM'

'Udondoshaji wa TJM ya shina'

'Msambao wa toni J'

'Uhusishaji wa Tonichini na Vitamkwa'

'Sharti la Ukubalifu'

'Umbo la nje' bé ryé ngé sya]. Matokeo ya ukokotozi (19) yanaonesha kwamba, ruwaza ya ujitokezaji wa toni 
katika kitenzi kisoukomo changamani [i mú bé ryé ngé sya], huanza kwa toni $\mathrm{C}$ katika kiambishi kisoukomo /i-/, ikifuatiwa na toni J katika mofimu ya yambwa na ambayo usambaa kuelekea kulia mwa shina hadi silabi ya mwisho kasoro moja na kuishia na toni $\mathrm{C}$ katika silabi ya mwisho ya shina. Aidha, katika ukokotozi wa (19), kanuni ya upachikaji wa TJM katika silabi ya kwanza ya shina na katika mofimu ya yambwa imetekelezwa kabla ya utekelezwaji wa kanuni ya udondoshaji wa TJM ya shina. Pia, kanuni ya msambao wa toni $\mathbf{J}$ kuelekea kulia mwa shina imetekelezwa kabla ya utekelezwaji wa Sharti la Ukubalifu katika hatua ya mwisho ya ukokotozi. Hebu, tuongeze mfano mmoja wa ukokotozi katika (20) hapa chini:

20.

\section{imúlémbéryílya}

/i mu le mbe ryi lya/

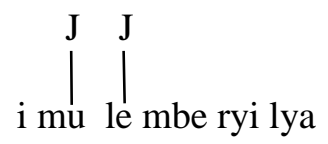

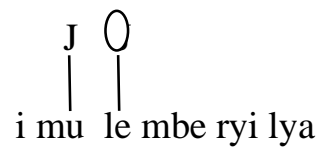

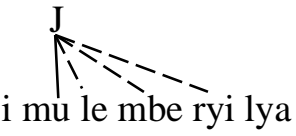<smiles>CC(C)(I)C1CCCCC1</smiles>

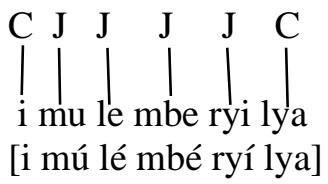

\section{'kumsingizia'}

'Umbo la ndani'

'Upachikaji wa TJM'

'Udondoshaji wa TJM ya shina'

'Msambao wa toni J'

'Uhusishaji wa Tonichini na Vitamkwa'
'Sharti la Ukubalifu'

'Umbo la nje'
Ukokotozi wa (20) hapo juu, umetupatia matokeo yanayokubalika katika usemaji wa Kirombo [i mú bé ryé ngé sya]. Matokeo ya ukokotozi (20) yanaonesha kwamba, ruwaza ya ujitokezaji wa toni katika kitenzi kisoukomo changamani [i mú bé ryé ngé sya], huanza kwa toni $\mathrm{C}$ katika kiambishi kisoukomo /i-/, ikifuatiwa na toni J katika mofimu ya yambwa na ambayo usambaa kuelekea kulia mwa shina hadi silabi ya mwisho kasoro moja na kuishia na toni $\mathrm{C}$ katika silabi ya mwisho ya shina. Aidha, katika ukokotozi wa (20), kanuni ya upachikaji wa TJM katika silabi ya kwanza ya shina na katika mofimu ya yambwa imetekelezwa kabla ya utekelezwaji wa kanuni ya udondoshaji wa TJM ya shina. Pia, kanuni ya msambao wa toni $\mathrm{J}$ kuelekea kulia mwa shina imetekelezwa kabla ya utekelezwaji wa Sharti la Ukubalifu katika hatua ya mwisho ya ukokotozi. Kulingana na matokeo ya ukokotozi katika (19-20) hapo juu, kukubalika katika usemaji wa Kirombo, basi, hatuna budi kuendelea na uchanganuzi wa kundi la pili la vitenzi visoukomo changamani lenye ruwaza ya ujitokezaji wa toni wa CJJJCC kama inavyoonekana katika data 21 hapa chini:
21. a) imúkándíkyao
'Kusiribia hapo'
b) imúkíshíkyao
'kufunikia hapo'
c) imútíngílyao
'Kuchechemea hapo'
d) imúfényílyao
'Kumenyea hapo'
e) imúféshíryao
'Kunong'onezea hapo

Katika data (21) hapo juu, vitenzi visoukomo changamani vina idadi sawa ya silabi za shina ambazo ni nne, mofimu moja ya yambwa /-m-/ na kiambishi kisoukomo/i-/ ambacho pia ni kiambishi awali cha vitenzi visoukomo vya Kirombo. Ukichunguza kwa makini vitenzi visoukomo changamani katika (21) utagundua kwamba 
muundo wa mfuatano wa silabi kuanzia kiambishi kisoukomo hadi silabi za shina hulandana na vyote vimeishia na irabu /-o/ mwishoni mwa shina. Irabu /-o/ ya mwisho wa shina hufanya kazi mbili: moja kiambishi tamati maana na mbili, hufanya kazi ya umahali 'hapo'. Pia, silabi ya mwisho kasoro moja kutoka mwishoni mwa shina la vitenzi visoukomo changamani vya (21), ina kiyeyusho ndani yake ambacho kimetokana na mchakato wa uyeyushaji. Aidha, kitoni vitenzi visoukomo changamani vya (21) vina toni C katika kiambishi kisoukomo /i-/, ikifuatiwa na toni $\mathrm{J}$ katika mofimu ya yambwa na ambayo usambaa kuelekea kulia hadi silabi ya pili ya shina na kisha kufuatiwa na toni $\mathrm{C}$, katika silabi ya mwisho kasoro moja na silabi ya mwisho ya shina la vitenzi husika pia. Ujitokezaji wa toni katika (21) ndio unaofanya kundi hili la pili la vitenzi visoukomo changamani vya silabi nne za shina kuwa na ruwaza ya CJJJCC. Ukokotozi wa uibuzi wa toni katika umbo la nje kutoka umbo la ndani la vitenzi visoukomo changamani katika data (21), utazingatia kanuni zilizotumika kukokotolea data ya (18) hapo juu. Hebu tuchunguze ukokotozi wa kitenzi kisoukomo changamani [imúkándíkyao] 'Kumsiribia hapo’ katika (22) hapa chini:

22.

$\begin{array}{ll}\text { imúkándíkyao } & \text { 'Kumsiribia hapo' } \\ \text { /i mu ka ndi kya o/ } & \text { 'Umbo la ndani' }\end{array}$

'Upachikaji wa TJM'

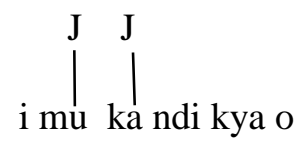

'Udondoshaji wa TJM ya shina'<smiles>CC(C)I</smiles>

i mu ka ndi kya o 'Msambao wa toni J'

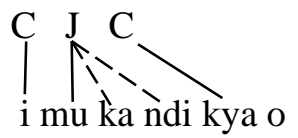

'Uhusishaji wa Tonichini na Vitamkwa'<smiles>COCC(I)(I)[AlH]I</smiles>

[i mú ká ndí kya o]
'Sharti la Ukubalifu'

'Umbo la nje'
Katika ukokotozi wa (22) hapo juu, tumepata matokeo yanayokubalika katika usemaji wa Kirombo [i mú ká ndí kya o]. Matokeo ya ukokotozi (22) yanaonesha kwamba, ruwaza ya ujitokezaji wa toni katika kitenzi kisoukomo changamani [i mú ká ndí kya o], huanza kwa toni $\mathrm{C}$ katika kiambishi kisoukomo /i-/, ikifuatiwa na toni J katika mofimu ya yambwa /-m-/ na ambayo usambaa kuelekea kulia mwa shina hadi silabi ya mwisho kasoro mbili na kufuatiwa na toni $\mathrm{C}$ katika silabi ya mwisho kasoro moja na katika silabi ya mwisho ya shina. Aidha, ukokotozi (22) umetumia kanuni mbalimbali ambapo kanuni ya jumla ya upachikaji wa TJM katika silabi ya kwanza ya shina na katika mofimu ya yambwa imetekelezwa katika hatua ya mwanzo ya ukokotozi. Pia, kanuni ya udondoshaji wa TJM ya shina imetekelezwa kabla ya utekelezwaji wa kanuni ya msambao wa toni $\mathbf{J}$ kuelekea kulia mwa shina, kishaa kufuatiwa na hatua ya uhusishaji wa tonichini na vitamkwa kaba ya utekelezwaji wa Sharti la Ukubalifu katika hatua ya mwisho ya ukokotozi wa uibuzi wa toni katika kitenzi kisoukomo changamani [i mú ká ndí kya o]. Hebu tuongeze mfano mmoja wa ukokotozi katika 23 hapa chini: 


$$
\begin{aligned}
& \text { imúkíshíkyao } \\
& \text { /i mu ki shi kya o/ }
\end{aligned}
$$

23.<smiles>O=[W][13CH](O)I</smiles><smiles></smiles>

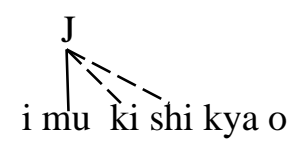

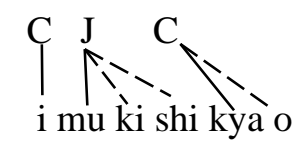<smiles>CO[AlH2]I(I)[AlH2]I</smiles>

[i mú kí shí kya o]
'Kumfunikia hapo'

'Umbo la ndani'

'Upachikaji wa TJM'

'Udondoshaji wa TJM ya shina'

'Msambao wa toni J'

'Uhusishaji wa Tonichini na Vitamkwa'

'Sharti la Ukubalifu'

'Umbo la nje'
Ukokotozi wa (23) hapo juu, umetupatia matokeo yanayokubalika katika usemaji wa Kirombo [i mú kí shí kya o]. Matokeo ya ukokotozi (23) yanaonesha kwamba, ruwaza ya ujitokezaji wa toni katika kitenzi kisoukomo changamani [i mú kí shí kya o], huanza kwa toni $\mathrm{C}$ katika kiambishi kisoukomo /i-/, ikifuatiwa na toni J katika mofimu ya yambwa /-m-/ na ambayo usambaa kuelekea kulia mwa shina hadi silabi ya mwisho kasoro mbili na kufuatiwa na toni $\mathrm{C}$ katika silabi ya mwisho kasoro moja na katika silabi ya mwisho ya shina. Aidha, ukokotozi (23) umetumia kanuni mbalimbali ambapo kanuni ya jumla ya upachikaji wa TJM katika silabi ya kwanza ya shina na katika mofimu ya yambwa imetekelezwa katika hatua ya mwanzo ya ukokotozi. Pia, kanuni ya udondoshaji wa TJM ya shina imetekelezwa kabla ya utekelezwaji wa kanuni ya msambao wa toni $\mathbf{J}$ kuelekea kulia mwa shina, kisha kufuatiwa na hatua ya uhusishaji wa tonichini na vitamkwa kabla ya utekelezwaji wa Sharti la Ukubalifu katika hatua ya mwisho ya ukokotozi wa uibuzi wa toni katika kitenzi kisoukomo changamani [i mú kí shí kya o]

Tofauti za ujitokezaji wa ruwaza ya toni katika makundi hayo mawili ya vitenzi visoukomo changamani kama yalivyojadiliwa katika data (21) na jinsi yalivyokokotolewa katika (22-23), ndiyo sababu zilizosababisha kuwa na makundi mawili ya ruwaza ya ujitokezaji wa toni katika vitenzi visoukomo changamani vya silabi nne za shina katika Kirombo. Katika makundi hayo mawili, vitenzi visoukomo changamani katika 18 (a-e) vina ruwaza ya ujitokezaji wa toni wa CJJJJC, wakati katika 21(a- e) vina ruwaza ya ujitokezaji wa toni wa CJJJCC. Kutokana na matokeo ya ukokotozi wa uibuzi wa toni katika makundi hayo mawili ya ujitokezaji wa ruwaza ya toni katika vitenzi visoukomo changamani vya silabi nne za shina, tunaweza kuendelea na mjadala wetu wa uchanganuzi wa ruwaza ya ujitokezaji wa toni katika vitenzi visoukomo changamani vya silabi tano za shina kama ifuatavyo:

\section{Ujitokezaji wa Ruwaza za Toni katika Vitenzi Visoukomo changamani vya Silabi Tano za Shina}

Baada ya kujadili ruwaza za ujitokezaji wa toni katika vitenzi visoukomo changamani vya silabi nne za shina, kwa kutalii makundi mawili ya CJJJJC na CJJJCC ambayo yalionekana kuwa na ruwaza tofauti za ujitokezaji wa toni katika vitenzi visoukomo changamani vya silabi nne za shina, kama yalivyofafanuliwa katika data (22) na (23) tumeona matokeo ya ukokotozi wake yanakubalika 
katika usemaji wa Kirombo. Hivyo, hatuna budi kuendelea kutalii vitenzi visoukomo changamani vya silabi tano za shina ambavyo kulingana na data ya utafiti vimebainika kuwa na makundi mawili ya ruwaza za ujitokezaji wa toni ambayo ni: CJJJJJC na CJJJCJC. Hebu tuchunguze data (24) hapa chini:

24. a) imúfáfásyíryílya

b) imútúkúlyíryílya

c) imútótónyíryílya

d) imúsúmányínyándu

e) imúsósófyíkyírya

f) imúsíkányíryírya

g) imútámbílyatábu

h) imúsúngúsyiryílya

i) imútámbúsyiryílya

j) imúdíshílyiryílya

k) imútóngólyiryílya

Katika data (24) hapo juu, vitenzi visoukomo changamani vyote vina idadi sawa ya silabi za shina ambazo ni tano na vina muundo wa mfuatano wa silabi unaolandana kwa kila kundi. Yaani, kila kundi lina mfuatano wake wa silabi za shina ambao ni tofauti na kundi jingine. Kwa mfano, kundi la kwanza katika 24(a-f) silabi zake za shina zimeanza kwa KI kuanzia silabi ya kwanza hadi silabi ya pili ya shina na kufuatia na kufuatiwa na KyI katika silabi ya tatu hadi silabi ya mwisho ya shina, wakati kundi la pili katika 24(g-k) lina KI katika silabi ya kwanza ya shina na kufuatiwa na KKI katika silabi ya pili ya shina, kisha kufuatiwa na KyI katika silabi ya tatu hadi silabi ya mwisho ya shina. Pia, vitenzi visoukomo changamani katika (24) vimeanza kwa kiambishi kisoukomo /i-/ na ambacho ni kiambishi awali cha vitenzi visoukomo vya Kirombo ambacho hufuatiwa na mofimu /-m-/ ya yambwa.

Kitoni, katika 24(a-f) kiambishi kisoukomo /i-/ kina toni C, ikifuatiwa na toni J katika mofimu /-m/ ya yambwa na ambayo usambaa kuelekea kulia mwa shina hadi silabi ya pili kasoro moja kutoka mwishoni mwa shina la kitenzi na kuishia na toni $\mathrm{C}$ katika silabi ya mwisho ya shina la vitenzi visoukomo changamani husika. Aidha, inaonekana dhahiri kuwa ruwaza ya ujitokezaji wa toni hubadilika kulingana na muundo wa aina ya kitenzi chenyewe kama inavyodhihirika katika 24(g- k) ambapo ruwaza ya ujitokezaji wa toni ni CJJJCJC, ambayo ni tofauti na ya kundi la kwanza. Yaani, katika kundi la 24(g-k) kiambishi kisoukomo /i-/ kina toni C, ikifuatiwa na toni J katika mofimu /-m/ ya yambwa na ambayo usambaa kuelekea kulia mwa shina hadi silabi ya pili kutoka mwanzoni mwa kitenzi, kwa kutatizwa na ujitokezaji wa toni C, katika silabi ya tatu ya shina. Aidha, toni J hujitokeza tena katika silabi ya pili kasoro moja kutoka mwishoni mwa shina la kitenzi na kuishia na toni $C$ katika silabi ya mwisho ya shina la vitenzi husika ambayo ni tabirifu. Kanuni ya unakiliji wa TJM ambayo imeundwa na kutumika katika data (24), imetumika kwa kuinakilisha katika silabi ya mwisho kasoro moja, pamoja na kanuni nyingine za toni katika kukokotoa data 24(g-k). Tuchunguze ukokotozi wa vitenzi visoukomo changamani vya kundi la kwanza la 24(a-f) katika 25 hapa chini:

\section{imúfáfásyíryílya}

/i mu fa fa syi ryi lya/

25.

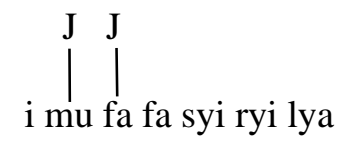

$$
\text { i mu fa fa syi ryi lya }
$$

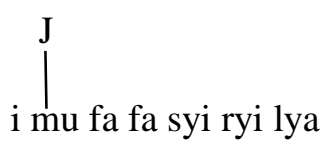

\section{'kumelekeza' \\ 'Umbo la ndani'}

'Upachikaji wa TJM'

'Udondoshaji wa TJM ya shina'

'Upachikaji wa TJM' 
<smiles>Ic1ccccc1</smiles>

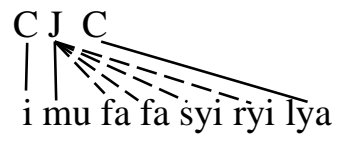

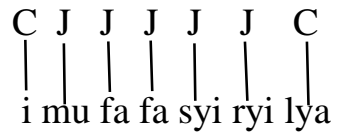

[i mú fá fá syí ryí lya]
'Usambaaji wa TJM'

'Uhusishaji wa Tonichini na Vitamkwa'

'Sharti la Ukubalifu'

'Umbo la nje'
Ukokotozi wa (25) hapo juu umetupatia matokeo yanayokubalika katika usemaji wa Kirombo [i mú fá fá syí ryí lya]. Matokeo ya ukokotozi wa 25 yanaonesha kuwa, ruwaza ya ujitokezaji wa toni katika umbo la nje kutoka umbo la ndani la kitenzi kisoukomo changamani huanza kwa toni $\mathrm{C}$ katika kiambishi kisoukomo /i-/, ikifuatiwa na toni $\mathbf{J}$ katika mofimu ya yambwa /-m-/ na ambayo usambaa kuelekea kulia mwa shina hadi silabi ya mwisho kasoro moja. Aidha, ukichunguza kwa makini ukokotozi (25) utagundua kwamba, kanuni ya jumla ya upachikaji wa TJM katika silabi ya kwanza ya shina imetekelezwa kabla ya utekelezwaji wa kanuni ya udondoshaji wa TJM ya shina. Pia, baada ya udondoshwaji wa TJM ya shina, kanuni ya msambao wa toni J kuelekea kulia mwa shina ilitekelezwa kabla ya utekelezwaji wa hatua ya uhusishaji wa tonichini na vitamkwa. Sharti la Ukubalifu limetekelezwa katika hatua ya mwisho ya ukokotozi wa kitenzi kisoukomo changamani [i mú fá fá syí ryí lya] kama inavyoonekana hapo juu. Hebu tuongeze mfano mmoja wa ukokotozi katika 26 hapa chini: imútúkúlyíryílya

/i mu tu ku lyi ryi lya/

26.

\section{'kumwangalia'}

'Umbo la ndani'

'Upachikaji wa TJM'

'Udondoshaji wa TJM ya shina'

'Upachikaji wa TJM'

'Usambaaji wa TJM'

'Sharti la Ukubalifu'

'Umbo la nje'
Ukokotozi wa 26 hapo juu umetupatia matokeo yanayokubalika katika usemaji wa Kirombo [i mú tú kú lyí ryí lya]. Matokeo ya ukokotozi wa 26 yanaonesha kuwa, ruwaza ya ujitokezaji wa toni katika umbo la nje kutoka umbo la ndani la kitenzi kisoukomo changamani huanza kwa toni $\mathrm{C}$ katika 
kiambishi kisoukomo /i-/, ikifuatiwa na toni $\mathbf{J}$ katika mofimu ya yambwa /-m-/ na amabyo usambaa kuelekea kulia mwa shina hadi silabi ya mwisho kasoro moja. Aidha, ukichunguza kwa makini ukokotozi 26 utagundua kwamba, kanuni ya jumla ya upachikaji wa TJM katika silabi ya kwanza ya shina imetekelezwa kabla ya utekelezwaji wa kanuni ya udondoshaji wa TJM ya shina. Pia, baada ya udondoshwaji wa TJM ya shina, kanuni ya msambao wa toni J kuelekea kulia mwa shina ilitekelezwa kabla ya utekelezwaji wa

imútámbílyatábu

/i mu ta mbi lya ta bu/

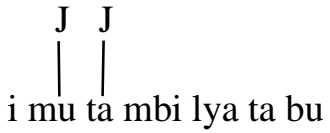<smiles>[14CH3][14CH2][14C@@H](O)I</smiles>

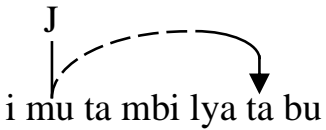

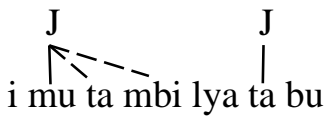

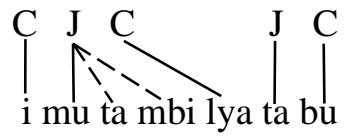

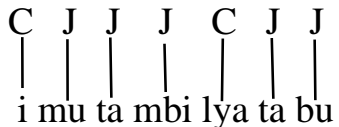

[i mú tá mbí lya tá bu] hatua ya uhusishaji wa tonichini na vitamkwa. Sharti la Ukubalifu limetekelezwa katika hatua ya mwisho ya ukokotozi wa kitenzi kisoukomo changamani [i mú tú kú lyí ryí lya] kama inavyoonekana hapo juu. Kulingana na matokeo ya ukokotozi wa uibuzi wa toni katika vitenzi visoukomo changamani katika 26(a-f) kukubalika katika usemaji wa Kirombo, basi hatuna budi kuendelea na uchanganuzi wa ruwaza ya kundi la pili katika 27 hapa chini.

27.

'kumng'nga'nia'

'Umbo la ndani'

'Upachikaji wa TJM'

'Udondoshaji wa TJM ya shina'

'Unakiliji wa TJM'

'Msambao wa toni J'

'Uhusishaji wa Tonichini na Vitamkwa'

'Sharti la Ukubalifu'

'Umbo la nje'

Katika ukokotozi (27) hapo juu, tumepata matokeo yanayokubalika katika usemaji wa Kirombo [i mú tá mbí lya tá bu]. Matokeo ya ukokotozi wa uibuzi wa toni katika umbo la nje kutoka umbo la ndani la kitenzi kisoukomo changamani [i mú tá mbí lya tá bu] (27) yanaonesha kwamba, ruwaza ya ujitokezaji wa toni huanza kwa toni $\mathrm{C}$ katika kiambishi kisoukomo /i-/, ikifuatiwa na toni $\mathrm{J}$ katika mofimu ya yambwa /-m-/ na ambayo imesambaa kuelekea kulia mwa shina hadi silabi ya tano ya shina na kuishia na toni $\mathrm{C}$ katika silabi ya mwisho ya shina. Aidha, katika ukokotozi wa (27) kanuni ya jumla ya upachikaji wa TJM katika silabi ya kwanza ya shina na katika mofimu ya yambwa imetekelezwa katika hatua ya mwanzo ya ukokotozi kabla ya utekelezwaji wa kanuni ya udondoshaji wa TJM ya shina. Pia, katika ukokotozi (27) hapo juu, kanuni ya unakiliji wa TJM katika silabi ya mwisho kasoro moja imetekelezwa kabla ya utekelezwaji wa kanuni ya msambao wa toni $\mathbf{J}$ kuelekea kulia mwa shina. Sanjari na hayo, hatua ya uhusishaji wa tonichini na vitamkwa imetekelezwa kabla ya utekelezwaji wa Sharti la Ukubalifu kama inavyodaiwa na Nadharia ya FVH. Hebu tuongeze ukokotozi mmoja katika 28 hapa chini kabla ya uchanganuzi wa ruwaza ya 
toni katika vitenzi visoukomo changamani vya silabi sita za shina.

$\begin{array}{ll}\text { imúsúngúsyiryílya } & \text { 'kumtengenezea } \\ \text { /i mu su ngu syi ryi lya/ } & \text { 'Umbo la ndani' }\end{array}$

28.

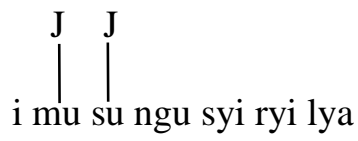

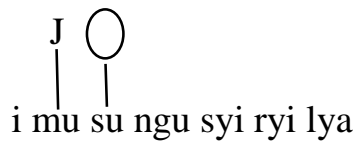<smiles></smiles><smiles>I[13CH]C1CC1I</smiles>

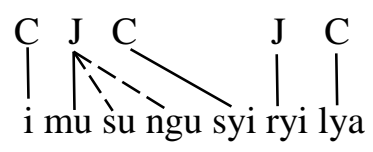<smiles>I[Al](I)[Si](I)(I)[Si](I)(I)[Al](I)I</smiles>

'Upachikaji wa TJM'

'Udondoshaji wa TJM ya shina'

'Unakiliji wa TJM'

'Msambao wa toni J'

'Uhusishaji wa Tonichini na Vitamkwa'

[i mú sú ngú syi ryí lya] 'Umbo la nje'

Katika ukokotozi (28) hapo juu, tumepata matokeo yanayokubalika katika usemaji wa Kirombo [i mú sú ngú syi ryí lya]. Matokeo ya ukokotozi wa uibuzi wa toni katika umbo la nje kutoka umbo la ndani la kitenzi kisoukomo changamani [i mú sú ngú syi ryí lya] katika (28) yanaonesha kwamba, ruwaza ya ujitokezaji wa toni huanza kwa toni $\mathrm{C}$ katika kiambishi kisoukomo /i-/, ikifuatiwa na toni $\mathrm{J}$ katika mofimu ya yambwa /-m-/ na ambayo imesambaa kuelekea kulia mwa shina hadi silabi ya tano ya shina na kuishia na toni $\mathrm{C}$ katika silabi ya mwisho ya shina. Aidha, katika ukokotozi wa (28), kanuni ya jumla ya upachikaji wa TJM katika silabi ya kwanza ya shina na katika mofimu ya yambwa imetekelezwa katika hatua ya mwanzo ya ukokotozi kabla ya utekelezwaji wa kanuni ya udondoshaji wa TJM ya shina ambayo ndiyo huruhusu kanuni ya msambao wa toni J kuelekea kulia mwa shina kufanya kazi. Pia, katika ukokotozi (28) hapo juu, kanuni ya unakiliji wa TJM katika silabi ya mwisho kasoro moja imetekelezwa kabla ya utekelezwaji wa kanuni ya msambao wa toni $\mathbf{J}$ kuelekea kulia mwa shina. Sanjari na hayo, hatua ya uhusishaji wa tonichini na vitamkwa imetekelezwa kabla ya utekelezwaji wa Sharti la Ukubalifu kama inavyodaiwa na Nadharia ya FVH. Kulingana na matokeo ya ukokotozi wa uibuzi wa toni katika umbo la nje kutoka umbo la ndani la makundi mawili ya ruwaza za toni katika vitenzi visoukomo changamani vya silabi tano za shina kuanzia katika (25-28), na ambayo hukubalika katika usemaji wa Kirombo, hatuna budi kuendelea na uchanganuzi wa ruwaza ya toni katika vitenzi visoukomo changamani vya silabi sita za shina katika sehemu inayofuata. 


\section{Ujitokezaji wa Ruwaza ya Toni katika Vitenzi Visoukomo Changamani vya Silabi Sita za Shina}

Baada ya kujadili kwa kina ruwaza za ujitokezaji wa toni katika vitenzi visoukomo changamani vya silabi tano za shina la kitenzi kwa kutumia kanuni mbalimbali za toni zikiwemo za unakiliji wa TJM na msambao wa toni $\mathbf{J}$ kuelekea kulia mwa shina la kitenzi na kanuni nyingine zilizozingatiwa katika uchanganuzi wa uibuzi wa toni kutoka umbo la ndani kwenda umbo la nje la vitenzi hivyo, tunaweza sasa kutalii ruwaza ya toni katika vitenzi visoukomo changamani vya silabi sita za shina. Kulingana na data ya utafiti, imebainika kuwa ruwaza ya ujitokezaji wa toni katika vitenzi visoukomo changamani vya silabi sita za shina ni CJJJJCJC. Chunguza data (29) hapa chini:

29.
a) imúdíshíryílyaséo
b) imúfáryísyíryaséo
c) imúkúshúryílyaséo
d) imúsúngúsyíryaséo
e) imúsóryómólyaséo
f) imútíryatíryaséo
g) imúdúmbúlyíryaséo
h) imútámbúkyíryaséo
i) imútámbúlyíryaséo

\author{
'kumkimbizia hapo' \\ 'kumzalishia hapo' \\ 'kumkumbatia hapo' \\ 'kumtengenezea hapo' \\ 'kumchomolea hapo \\ 'kumuogopeshea mahali fulani' \\ 'kumkatia sehemu fulani' \\ 'kumtembelea hapo' \\ 'kumnusuru hapo'
}

Ukichunguza kwa makini data (29) hapo juu, utagundua kwamba kimofolojia vitenzi visoukomo changamani vyote vina silabi sita za shina ambazo kwa kiasi kikubwa ulandana kwa namna moja au nyingine kimuundo. Vitenzi visoukomo changamani katika (29) vimeanza kwa kiambishi awali /i-/ ambacho pia ni kisoukomo cha vitenzi visoukomo vya Kirombo na kufuatiwa na mofimu ya yambwa /-m-/, kisha silabi ya kwanza ya shina yenye muundo wa KI. Pia, vitenzi vyote katika (29) vimeishia kwa irabu /-o/ ambayo hufanya kazi kama kiambishi tamati na kiangami cha mahali ambapo tendo hutendekea. Aidha, kitoni, vitenzi visoukomo changamani katika (29) vina toni J tano katika silabi za shina na toni $\mathrm{J}$ moja katika mofimu ya yambwa. Pia, vina toni $\mathrm{C}$ mbili za shina: moja katika silabi ya mwisho kasoro mbili na ya pili katika silabi ya mwisho ya shina. Pia, vitenzi visoukomo changamani katika (29) vina toni C katika kiambishi kisoukomo /i-/. Ujitokezaji wa toni katika data (29) ndio unaofanya kundi la vitenzi visoukomo changamani vya silabi sita za shina kuwa na ruwaza ya ujitokezaji wa toni wa CJJJJCJC. Yaani, ruwaza ya toni katika (29) imeanza kwa toni C katika kiambishi kisoukomo /i/, ikifuatiwa na toni J katika mofimu ya yambwa /m-/ na ambayo usambaa kuelekea kulia mwa shina hadi silabi ya tatu ya shina ambapo hutatizwa kwa kujitokeza toni $\mathrm{C}$ katika silabi ya nne ya shina, ambayo ujitokeza katikati ya shina na ambayo hufuatiwa na toni J katika silabi ya tano ya shina na kuishia na toni $\mathrm{C}$ katika silabi ya mwisho ya shina la vitenzi husika.

Pia, ukichunguza kwa makini ruwaza ya ujitokezaji wa toni katika 171 ambayo ni CJJJJCJC utagundua kwamba, ina TJM mbili kama ilivyokuwa kwa vitenzi vya silabi tano za shina katika data 24 (g-k) ambayo tulilazimika kutumia kanuni ya unakiliji wa TJM. Kwa kuwa data ya 29 nayo ina TJM mbili basi kanuni ya 7 ya unakiliji wa TJM imetumika katika ukokotozi wa uibuzi wa toni katika umbo la nje kutoka umbo la ndani katika 30 hapa chini: imúdíshíryílyaséo

/i mu di shi ryi lya se o/

30.

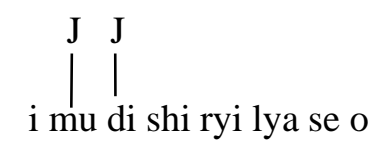

'kumkimbizia hapo'

'Umbo la ndani'

'Upachikaji wa TJM' 


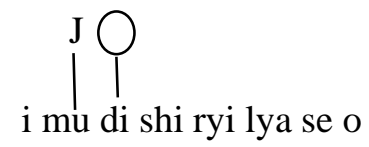

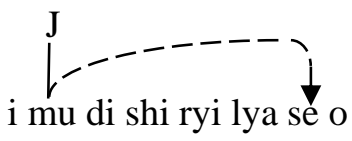

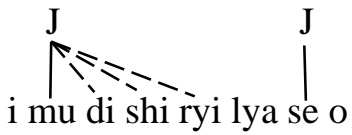

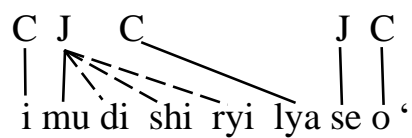

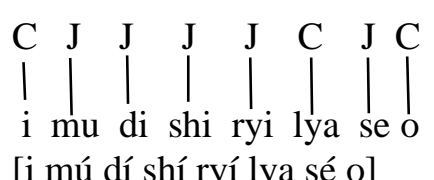

'Udondoshaji wa TJM ya shina'

'Unakiliji wa TJM'

'Msambao wa toni J'
'Sharti la Ukubalifu'

'Umbo la nje'
Katika ukokotozi (30) hapo juu, tumepata matokeo yanayokubalika katika usemaji wa Kirombo [i mú dí shí ryí lya sé o]. Matokeo yanaonesha kwamba, ruwaza ya ujitokezaji wa toni katika umbo la nje kutoka umbo la ndani la kitenzi kisoukomo changamani [i mú dí shí ryí lya sé o] ni CJJJJCJC. Yaani, huanza kwa toni $\mathrm{C}$ katika kiambishi kisoukomo /i-/, ikifuatiwa na toni J katika mofimu ya yambwa /-m-/ na ambayo usambaa kuelekea kulia mwa shina hadi silabi ya tatu ambapo huzuiliwa na toni $\mathrm{C}$ ambayo ujitokeza katika silabi ya nne katikati ya shina, kisha kufuatiwa na toni $\mathbf{J}$ ambayo hujitokeza katika silabi ya tano na kuishia na toni $C$ katika silabi ya mwisho ya shina. Ukichunguza kwa makini ukokotozi (30) utagundua kwamba, kanuni ya jumla ya upachikaji wa TJM katika silabi ya kwanza ya shina na katika mofimu ya yambwa /-m-/ imetekelezwa katika hatua ya mwanzo ya ukokotozi. Pia, kanuni ya udondoshaji wa TJM ya shina imetekelezwa kabla ya utekelezwaji wa kanuni ya unakiliji wa TJM katika silabi ya tano ya shina. Sanjari na hilo, kanuni ya msambao wa toni $\mathbf{J}$ imetekelezwa kabla ya utekelezwaji wa hatua ya uhusishaji wa tonichini na vitamkwa ambayo imefuatiwa na utekelezwaji wa Sharti la Ukubalifu kama inavyoelezwa katika Nadharia ya FVH katika hatua ya mwisho ya ukokotozi wa kitenzi kisoukomo changamani [i mú dí shí ryí lya sé o]. Kabla ya kuendelea na uchanganuzi wa vitenzi visoukomo changamani vya silabi moja ya shina, tunaweza kuongeza ukokotozi mmoja wa vitenzi visoukomo changamani vya silabi sita za shina katika 31 hapa chini: imúfáryísyíryaséo

/i mu fa ryi syi rya se o/

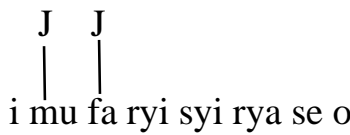

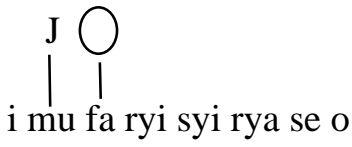

'kumzalishia hapo'

'Umbo la ndani'

'Upachikaji wa TJM'

'Udondoshaji wa TJM ya shina' 


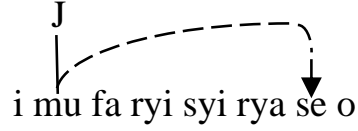

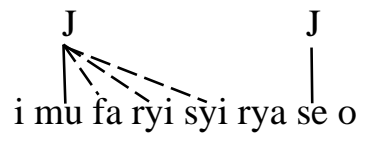

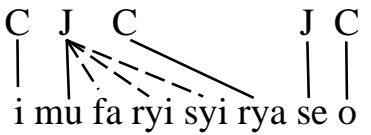

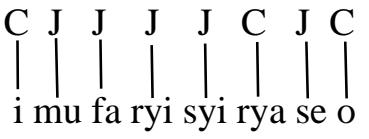

'Unakiliji wa TJM'

'Msambao wa toni J'

'Uhusishaji wa Tonichini na Vitamkwa

'Sharti la Ukubalifu'

'Umbo la nje'
Ukokotozi wa (31) hapo juu, umetupatia matokeo yanayokubalika katika usemaji wa Kirombo [i mú fá ryí syí rya sé o]. Matokeo yanaonesha kwamba, ruwaza ya ujitokezaji wa toni katika umbo la nje kutoka umbo la ndani la kitenzi kisoukomo changamani [i mú fá ryí syí rya sé o] ni CJJJJCJC. Yaani, huanza kwa toni $\mathrm{C}$ katika kiambishi kisoukomo /i-/, ikifuatiwa na toni J katika mofimu ya yambwa /-m-/ na ambayo usambaa kuelekea kulia mwa shina hadi silabi ya tatu ambapo huzuiliwa na toni $\mathrm{C}$ ambayo ujitokeza katika silabi ya nne katikati ya shina, kisha kufuatiwa na toni $\mathbf{J}$ ambayo hujitokeza katika silabi ya tano na kuishia na toni $\mathrm{C}$ katika silabi ya mwisho ya shina. Ukichunguza kwa makini ukokotozi (31) utagundua kwamba, kanuni ya jumla ya upachikaji wa TJM katika silabi ya kwanza ya shina na katika mofimu ya yambwa /-m-/ imetekelezwa katika hatua ya mwanzo ya ukokotozi. Pia, kanuni ya udondoshaji wa TJM ya shina imetekelezwa kabla ya utekelezwaji wa kanuni ya unakiliji wa TJM katika silabi ya tano ya shina. Sanjari na hilo, kanuni ya msambao wa toni $\mathrm{J}$ imetekelezwa kabla ya utekelezwaji wa hatua ya uhusishaji wa tonichini na vitamkwa ambayo imefuatiwa na utekelezwaji wa Sharti la Ukubalifu kama inavyoelezwa katika Nadharia ya FVH katika hatua ya mwisho ya ukokotozi wa kitenzi kisoukomo changamani [i mú fá ryí syí rya sé o].

Kutokana na matokeo ya ukokotozi wa data $31 \mathrm{ni}$ wazi kwamba uibuzi wa ruwaza ya ujitokezaji wa toni katika vitenzi changamani vya silabi mbili hadi vile vya silabi sita hausigani. Aidha, ukichunguza kwa makini namna ukokotozi wake ulivyofanywa utagundua kuwa ruwaza ya ujitokezaji wa toni katika vitenzi visoukomo changamani huanza na $\mathrm{C}$ katika kiambishi kisoukomo /i-/, ikifuatiwa na toni J inayojitokeza katika mofimu inayowakilisha yambwa. Pia, tumeona kwamba, silabi ya kwanza ya shina nayo huwa na toni $\mathrm{J}$ ambayo baadae udondoshwa ili kuruhusu kanuni ya msambao wa toni J kuelekea kulia iweze kufanya kazi. Msambao huo wa toni J kuelekea kulia mwa shina umeenda hadi silabi ya mwisho kasoro moja kutoka mwishoni mwa shina la vitenzi husika. Silabi ya mwisho ya shina la vitenzi visoukomo changamani vya silabi mbili hadi sita vina $\mathrm{C}$ ambayo ni tabirifu.

\section{Ujitokezaji wa Ruwaza ya Toni katika Vitenzi Visoukomo Changamani vya Silabi Moja ya Shina}

Baada ya kuona hali ya uchanganuzi wa toni katika vitenzi visoukomo changamani vya idadi tofauti ya silabi za shina kuanzia vile vya silabi mbili hadi silabi sita za shina, basi hatuna budi kuangazia uchanganuzi wa ruwaza ya ujitokezaji wa toni katika vitenzi visoukomo changamani vya silabi moja ya shina. Vitenzi hivi visoukomo changamani vya silabi moja vimeshughulikiwa mwishoni kulingana na upekee wa ruwaza yake ya ujitokezaji wa toni. Unaweza kuchunguza data (32) kama inavyoonekana hapa chini:
32. a)..i mú la
b) i mú lya
'kumuita'
c) i mú ma
'kumla'
d) i mú ra
'kummaliza'
e) i mú dwa
'kumpanda 


\section{f) i mú nya 'kumnya'}

Tukichunguza data (32) kwa makini tunaweza kuona mambo yafuatayo: Vitenzi visoukomo changamani vya silabi moja ya shina vina kiambishi awali $\{i-\}$ ambacho hufanya kazi ya usoukomo wa vitenzi ukomo vya Kirombo, vina mofimu \{-mu-\} ambayo huwakilisha yambwa na vina silabi moja ya shina. Aidha, inapaswa ifahamike kuwa muundo wa kitenzi cha silabi moja unaweza kuwa na konsonanti zaidi ya moja bila kuwa na shada la irabu ambalo kwa baadhi ya Lugha za Kibantu kikiwemo Kirombo huyeyusha irabu mojawapo au kuidondosha kama baadhi ya vitenzi vinavyoonekana katika 32(b, e na f). Pia, katika data (32) inaonekana kuwa KA imebeba toni $\mathrm{C}$ ikifuatiwa na toni $\mathrm{J}$ inayojitokeza katika mofimu $\{-\mathrm{mu}-\}$ ya yambwa na silabi ya shina huhusishwa na toni $\mathrm{C}$ ambayo ni tabirifu. Hali ya ujitokezaji wa toni katika (32) ndiyo inayofanya ruwaza ya ujitokezaji wa toni katika vitenzi visoukomo changamani vya silabi moja ya shina kuwa CJC. Katika ruwaza ya utokeaji wa toni katika vitenzi visoukomo changamani vya silabi moja ya shina toni $\mathbf{J}$ hujitokeza katika mofimu ya yambwa kama inavyoonekana katika data (181) hapo juu..

Hapa yafaa ikumbukwe mwanzo wa uchanganuzi wa vitenzi visoukomo changamani vya silabi mbili hadi sita za shina tulisema kuwa, kwa kuwa toni J hujitokeza katika mofimu inayowakilisha yambwa, na kwa kuwa TJM ya yambwa ina nguvu kuliko ile ya shina, basi, TJM ya shina udondoshwa. Hivyo, katika uchanganuzi wa vitenzi visoukomo changamani vya silabi moja ya shina, TJM ipachikwe kwenye mofimu ya yambwa na katika silabi ya shina kisha TJM ya silabi ya shina idondoshwe. Kanuni ya udondoshaji hutekelezwa baada ya upachikaji wa TJM na kabla ya utekelezaji wa hatua ya uhusishaji wa Tonichini na Vitamkwa. Hebu tuchunguze mfano wa ukokotozi wa data (32) katika (33) hapa chini:

33.

$\begin{array}{ll}\text { i mú ra } & \text { 'kumshika' } \\ \text { /i mu ra/ } & \text { 'Umbo la nje' }\end{array}$

'Upachikaji wa TJM'<smiles>O[AlH2]</smiles>

'Udondoshaji wa TJM ya shina'

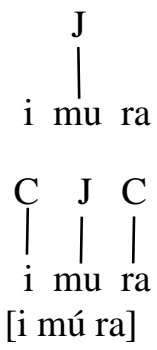

'Upachikaji wa TJM'

'Uhusishaji wa Tonichini na Vitamkwa'

'Umbo la nje'

Ukichunguza ukokotozi (33) kwa makini utagundua kwamba, umetupatia matokeo yanayokubalika katika usemaji wa wazungumzaji wazawa wa Kirombo [i mú ra]. Matokeo ya ukokotozi (33) yanaonesha kuwa, ruwaza ya ujitokezaji wa toni katika kitenzi [i mú ra] huanza kwa toni C katika KA, ikifuatiwa na toni J katika mofimu $\{-\mathrm{mu}-\}$ ya yambwa na kuishia na toni $\mathrm{C}$ katika silabi ya shina. Aidha, katika ukokotozi wa (33) TJM msingi zote za yambwa na shina zimehusishwa na kitamkwa husika kabla ya TJM ya shina kudondoshwa. Aidha, utagundua kuwa, kanuni ya udondoshaji wa TJM ya shina imetekelezwa baada ya utekelezaji wa kanuni ya jumla ya upachikaji wa TJM katika mofimu ya yambwa na katika silabi ya shina na kabla ya hatua ya uhusishaji wa Tonichini na Vitamkwa. Pia, katika ukokotozi (33) hakuna haja ya kutekeleza sharti la Ukubalifu kwa kuwa tayari limekwisha tekelezwa katika hatua ya Uhusishaji wa Tonichini 
na Vitamkwa. Tuongeze mfano mmoja wa ukokotozi wa uibuzi wa toni katika umbo la nje kutoka umbo la ndani la vitenzi visoukomo changamani vya silabi moja ya shina katika (34) hapa chini

$$
\begin{array}{ll}
\text { i mú la } & \text { 'Kumwita' } \\
\text { /i mu la/ } & \text { 'Umbo la ndani' }
\end{array}
$$

34.

$$
\left.\right|_{\mathrm{i} \text { mu la }} ^{\mathrm{J}} \mathrm{J}
$$<smiles>O[AlH2]O</smiles><smiles>I[13IH]I</smiles><smiles>C[AlH]I</smiles>
[i mú la]
'Upachikaji wa TJM'

'Udondoshaji wa TJM ya shina'

'Upachikaji wa TJM'

'Uhusishaji wa Tonichini na Vitamkwa'

'Umbo la nje' kama ilivyokuwa kwa mfano wa ukokotozi wa data (34), mfano wa ukokotozi wa data (34) nao pia, umetupatia matokeo yanayokubalika katika usemaji wa wazungumzaji wa Kirombo [i mú la]. Matokeo ya ukokotozi (34) yanaonesha kuwa, ruwaza ya ujitokezaji wa toni katika kitenzi [[i mú la] huanza kwa toni $\mathrm{C}$ katika KA, ikifuatiwa na toni J katika mofimu $\{-\mathrm{mu}-\}$ ya yambwa na kuishia na toni $\mathrm{C}$ katika silabi ya shina. Aidha, katika ukokotozi wa (34) TJM ya yambwa na shina zimehusishwa na kitamkwa husika kabla ya TJM ya shina kudondoshwa. Aidha, utagundua kuwa, kanuni ya udondoshaji wa TJM ya shina imetekelezwa baada ya utekelezaji wa kanuni ya jumla ya upachikaji wa TJM katika mofimu ya yambwa na katika silabi ya shina na kabla ya hatua ya uhusishaji wa Tonichini na Vitamkwa. Pia, katika ukokotozi (34) hakuna haja ya kutekeleza sharti la Ukubalifu kwa kuwa tayari limekwisha tekelezwa katika hatua ya Uhusishaji wa Tonichini na Vitamkwa.

Hata hivyo, badala ya kutumia hatua nyingi za ukokotozi katika kufikia matokeo yanayokubalika katika lugha ya wazungumzaji wazawa wa Kirombo, tunapendekeza kutumia njia mbadala ambayo itawawia rahisi wasomaji na mwanazuoni chipukizi katika masuala ya toni hususani toni katika Kirombo kwa kuchukulia kwamba, TJM inapaswa kupachikwa kwenye mofimu ya yambwa (Y) kwa vitenzi visoukomo changamani vya silabi moja ya shina katika Kirombo. Hebu tuchunguze mfano wa ukokotozi katika (35) hapa chini: $\begin{array}{ll}\text { i mú lya } & \text { 'kumla' } \\ \text { /i m lyal } & \text { 'Umbo la ndani' }\end{array}$

35. $\quad$ i mu lya

'Upachikaji wa TJM'

† $\left.\right|_{\text {i mu lya }} ^{\mathrm{J}}$

[i mú lya]

'Uhusishaji wa Tonichini na Vitamkwa' 
Ukokotozi wa data (35) umetupatia matokeo yanayokubalika katika usemaji wa wazungumzaji wa Kirombo [i mú lya]. Matokeo ya ukokotozi (35) yanaonesha kwamba, ruwaza ya ujitokezaji wa toni katika kitenzi [i mú lya] huanza kwa toni $\mathrm{C}$ katika $\mathrm{KA}$, ikifuatiwa na toni J katika mofimu $\{-\mathrm{mu}-\}$ ya yambwa na kuishia na toni $\mathrm{C}$ katika silabi ya shina. Ukokotozi wa (35) umefanyika kwa kufuata hatua mbili pekee ukilinganisha na ukokotozi wa data (33 na 34). Katika hatua ya kwanza, kanuni ya jumla ya
Upachikaji wa TJM imetekelezwa na kufuatiwa na hatua ya Uhusishaji wa Tonichini na Vitamkwa ambayo ni hatua ya pili. Aidha, kama ilivyokuwa kwa mfano wa ukokotozi wa data (33 na 34), ukokotozi wa data (35) nao pia, hauhitajii utekelezaji wa Sharti la Ukubalifu kwa kuwa limeshatekelezwa katika hatua ya pili kama inavyoonekana hapo juu. Tuongeze mfano mmoja wa ukokotozi katika (36) hapa chini:

$\begin{array}{ll}\text { i ú ma } & \text { 'kuumaliza' } \\ \text { /i u ma/ } & \text { 'Umbo la ndani' }\end{array}$

36.

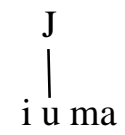

'Upachikaji wa TJM'

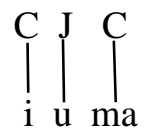

[i ú ma]

\section{'Uhusishaji wa Tonichini na Vitamkwa' \\ 'Umbo la nje'}

Ukokotozi wa data (36) umetupatia matokeo sahihi kulingana na wazungumzaji wa Kirombo [i ú ma]. Matokeo ya ukokotozi (36) yanaonesha kwamba, ruwaza ya ujitokezaji wa toni katika kitenzi [i ú ma] huanza kwa toni $\mathrm{C}$ katika KA, ikifuatiwa na toni $\mathrm{J}$ katika mofimu $\{-\mathrm{mu}-\}$ ya yambwa na kuishia na toni C katika silabi ya shina. Ukokotozi wa (36) umefanyika kwa kufuata hatua mbili pekee kama ukokotozi wa data (35). Katika hatua ya kwanza, kanuni ya jumla ya Upachikaji wa TJM imetekelezwa na kufuatiwa na hatua ya Uhusishaji wa Tonichini na Vitamkwa ambayo ni hatua ya pili. Aidha, kama ilivyokuwa kwa mfano wa ukokotozi wa data (35), ukokotozi wa data (36) nao pia, hauhitajii utekelezaji wa Sharti la Ukubalifu kwa kuwa limeshatekelezwa katika hatua ya pili kama inavyoonekana hapo juu.

\section{HITIMISHO}

Kwa hakika, tunaweza kuhitimisha kwa kusema kuwa, katika vitenzi visoukomo changamani vya silabi moja ya shina TJM hupachikwa katika mofimu $\{-\mathrm{mu}-\}$ inayowakilisha yambwa. Pia, kwa vile toni J ya yambwa ina nguvu zaidi basi, ile toni J ya shina udondoshwa kabla ya utekelezaji wa hatua ya Uhusishaji wa Tonichini na Vitamkwa.
Aidha, kwa ujumla ruwaza za ujitokezaji wa toni kwa vitenzi visoukomo changamani vya idadi ya silabi kuanzia moja hadi sita haisigani, kwa kuwa vitenzi vyote huanza na toni $\mathrm{C}$ katika $\mathrm{KA}$ ikifuatiwa na toni $\mathbf{J}$ ambayo usambaa hadi silabi ya mwisho kasoro moja kutoka mwishoni mwa shina la vitenzi husika na silabi ya mwisho wa shina huwa na toni $\mathrm{C}$ ambayo ni tabirifu. Pia kundi la vitenzi vya silabi mbili hadi sita hudondosha TJM ya shina si tu kwa vile TJM ya $\mathrm{Y}$ ina nguvu zaidi, bali pia kuruhusu kanuni ya msambao wa toni J kuelekea kulia mwa shina iweze kufanya kazi katika kufikia matokeo sahihi ya ujitokezaji wa ruwaza ya toni kwa wazungumzaji wa Kirombo.

Sanjari na hayo, tumebaini kuwa ruwaza za ujitokezaji wa toni katika vitenzi visoukomo changamani vya Kirombo zina muundo tofauti ambao hutawaliwa na kanuni ya msingi kisha kufuatiwa na kanuni nyingine za toni katika ukokotozi wa uibuzi wa toni katika umbo la nje kutoka umbo la ndani la vitenzi vya silabi moja hadi silabi sita za shina. Aidha, ruwaza ya ujitokezaji wa toni katika vitenzi visoukomo changamani vya silabi mbili hadi sita pamoja na kanuni nyingine kitoni hutawaliwa na kanuni ya msingi ya Udondoshaji wa TJM ya shina. Hata hivyo, ruwaza ya ujitokezaji wa toni katika vitenzi visoukomo changamani vya silabi moja ya shina ni CJC. Hii ni 
kwa sababu toni $\mathbf{J}$ hujitokeza katika mofimu inayowakilisha yambwa katika Kirombo.

\section{MAREJELEO}

Batibo, H. M. (2012), Can tone shift be regarded as tone depression in Shisukuma? Katika M. Brenzinger and A.-M. Fehn (Wah.), Proceedings of the $6^{\text {th }}$ World Congress of African Linguistics. kur. 201-206. Cologne: Ruediger Koeppe Verlag.

Goldsmith, J. (1976), “Autosegmental Phonology”. Tasnifu ya Uzamivu, MIT. (Imechapishwa).

Heine, B \& Nurse, D. (2003), (Wah.). African Languages: An Introduction. Cambridge: Cambridge University Press.

Hyman, L. M. (2003), Basaá (A43). Katika. Nurse \& Philippson, 257-282.

Hyman, L. M. (2008), Directional asymmetries in the morphology and phonology of words, with special reference to Bantu. Linguistics 46. 309350.

Hyman, L. M. (2017), Katika. search of prosodic domains in Lusoga. Paper presented at the Workshop on the Effects of Constituency in on Sentence Phonology, University of Massachusetts, Amherst, Julai 30, 2016. Ms. University of California, Berkeley (submitted).

Marlo, M. R. \& Odden, D. (2014), Bakweri tone melodies. Africana Linguistica 20.295-312.

Martinet, A. (2005), Economie des changements linguistiques. Paris: Maisonneuve et Larousse.

Massamba, D. P. B. (2011), Maendeleo katika Nadharia ya Fonolojia. Dar es Salaam: TATAKI.

Massamba, D. P. B. (2012), Misingi ya Fonolojia: Dar es Salaam. TATAKI.

Massamba, D. P. B. (2016), Kamusi ya Isimu na Falsafa ya Lugha. Dar es Salaam: TATAKI.

McHugh, B. D. (2006), The phrasal cycle in Kivunjo Chaga tonology. Katika. Inkelas \& Zec, kur. 217-242.
Meeussen, A. E. (1980), Bantu lexical reconstructions. Tervuren: Musée Royal de l'Afrique.

MLUTA. (2009), http/www.google.com/lugha/. LOT (10/4/2018).

Möller, K. (2014). Vom naturwissenschaftlichen Sachunterricht zum Fachunterricht-Der Übergang von der Grundschule in die weiterführende Schule. Zeitschrift für Didaktik der Naturwissenschaften, 20(1), 33-43.

Mramba, P. T. (2015), 'Athari za Kirombo katika ujifunzaji wa Kiswahili kama Lugha ya Pili: vipengele vya Kifonolojia na Kimofolojia'. Tasnifu ya Umahiri, Chuo Kikuu cha Dar es Salaam. (Haijachapishwa).

Nash, J. A. (1992), Underlying low tones in Ruwund. Studies in African Linguistics 23.223278.

Nelson, C. (2013), 'Toni katika Vitenzi vya Kimochi'. Tasnifu ya Umahiri, Chuo Kikuu cha Dar es Salaam. (Haijachapishwa).

Nurse, D \& Phillipson, G. (1977), “Tones in Old Moshi (Chaga)", katika Studies in African Linguistics 8, kur. 49-80.

Nurse, D. \& Philippson, G. (Wah.) (2003), The Bantu languages. London: Routledge.

Odden, D. (2005), Introducing Phonology. Cambridge: Cambridge University Press.

Polomé, S. A. (1971), 'Phonological Survey of the Chaga Dialect of Tanzania'. Tasnifu ya Umahiri, Austin Texas. (Imechapishwa).

Raum, J. (1964), Versuch einer Grammatik der Dschaggasprache (Moschi-Dialekt). U.S.A: The Gregg Press Incorporated.

Snider, K. (1999), On establishing underlying tonal contrast. Language Documentation and Conservation 8, kur. 669-699.

Stevick, E. W. (1969), Tone in Bantu. International Journal of American Linguistics 35.330-341. 
East African Journal of Swahili Studies, Volume 2, Issue 2, 2020

Article DOI: https://doi.org/10.37284/eajss.2.2.242

Van Spaandock, M. (1971), L'Analyse Morphotonologique dans les Langues Bantoues. Paris: SELAF. 